\title{
On the Eigenfunctions for the Multi-species q-Boson System
}

\author{
By \\ Yoshihiro TAKEYAMA \\ (University of Tsukuba, Japan)
}

\begin{abstract}
In a previous paper a multi-species version of the $q$-Boson stochastic particle system is introduced and the eigenfunctions of its backward generator are constructed by using a representation of the Hecke algebra. In this article we prove a formula which expresses the eigenfunctions by means of the $q$-deformed bosonic operators, which are constructed from the $L$-operator of higher rank found in the recent work by Garbali, de Gier and Wheeler. The $L$-operator is obtained from the universal $R$-matrix of the quantum affine algebra of type $A_{r}^{(1)}$ by the use of the $q$-oscillator representation. Thus our formula may be regarded as a bridge between two approaches to studying integrable stochastic systems by means of the quantum affine algebra and the affine Hecke algebra.

Key Words and Phrases. Affine Hecke algebra, Quantum group, Integrable system, Stochastic process.

2010 Mathematics Subject Classification Numbers. 17B80, 81R12, 60K35.
\end{abstract}

\section{Introduction}

In this article we prove a formula which expresses the eigenfunctions of the backward generator of a multi-species version of the $q$-Boson system (which is constructed in the previous paper [10]) by means of $q$-deformed bosonic operators.

The $q$-Boson system is a stochastic particle system on the one-dimensional discrete space with continuous time [9]. It is a zero-range process for bosonic particles, where one particle may hop to the left nearest-neighboring site independently for each site. It is integrable in the following sense. There exists the $L$-operator whose entries are $q$-deformed bosonic operators and the transfer matrix, which is the trace of a product of the $L$-operators, generates mutually commuting operators, one of which is the transition rate matrix of the $q$-Boson system with periodic boundary condition.

A multi-species version of the $q$-Boson system is introduced in [10]. It is also a zero-range process for bosonic particles, though each particle is colored with a positive integer and the transition rate depends on the color. As we will see in Section 4.3 below, using the $L$-operator of higher rank found in the recent work of Garbali, de Gier and Wheeler [4], we can construct a family of commuting operators which includes the transition rate matrix of the multi- 
species $q$-Boson system with periodic boundary. In this sense the system is also integrable. Recently it has been clarified that it is related to a representation theory of the quantum affine algebra $[6,7]$.

Originally, the multi-species $q$-Boson system was constructed from a representation of a deformation of the affine Hecke algebra of type GL. In this algebraic setting we can easily construct as a byproduct the eigenfunctions of the backward generator of the system. They are expressed by means of an action of the Hecke algebra of type $A_{k-1}$ on the vector space $\left(\boldsymbol{C}^{r}\right)^{\otimes k}$, where $k$ is the total number of particles and $r$ is the number of species of particles. When $r=1$ they are equal to the eigenfunctions found by Borodin, Corwin, Petrov and Sasamoto [2]. A similar formula for the configuration probability in the multi-species asymmetric exclusion process is obtained by Tracy and Widom [11].

The purpose of this paper is to prove a formula which expresses the eigenfunctions for the multi-species $q$-Boson system by means of $q$-deformed bosonic operators. The formula is of the following form

$$
\lim _{\substack{M \rightarrow \infty \\ M^{\prime} \rightarrow-\infty}} \prod_{i=1}^{k} \frac{z_{i}^{M^{\prime}-1}}{\left(1+z_{i}\right)^{M}}\left\langle\prod_{1 \leq i \leq k}^{\curvearrowright} C_{\mu_{i}}^{\left[M^{\prime}, M\right]}\left(z_{i}\right) \prod_{1 \leq i \leq k} \beta_{v_{i}, x_{i}}^{*}\right\rangle_{\left[M^{\prime}, M\right]} .
$$

It is a function on the set of configurations of $k$ particles with $r$ species on $\boldsymbol{Z}$, and $\left(x_{1}, \ldots, x_{k}\right)$ and $\left(v_{1}, \ldots, v_{k}\right)$ signify the positions and the colors of the particles, respectively. The operator $C_{a}^{\left[M^{\prime}, M\right]}(z)(1 \leq a \leq r)$ is a $q$-deformed bosonic operator produced from an ordered product of the $L$-operators assigned to the sites on the interval $\left[M^{\prime}, M\right] \subset \boldsymbol{Z}$, and $\beta_{a, i}^{*}$ is the creation operator assigned to the $i$-th site. The eigenfunctions are parametrized by two tuples $\left(z_{1}, \ldots, z_{k}\right) \in C^{k}$ and $\left(\mu_{1}, \ldots, \mu_{k}\right) \in\{1,2, \ldots, r\}^{k}$ such that the number of $\mu_{i}$ 's satisfying $\mu_{i}=a$ is equal to that of particles with color $a(1 \leq a \leq r)$. The symbol \langle\rangle$_{\left[M^{\prime}, M\right]}$ represents the matrix element between the vacuum vector and its dual on the interval $\left[M^{\prime}, M\right]$.

As shown in [4], the $L$-operator of higher rank, which is a source of the $q$-deformed bosonic operator $C_{a}^{\left[M^{\prime}, M\right]}(z)$, can be constructed from the universal $R$-matrix of the quantum affine algebra of type $A_{r}^{(1)}$ by the use of the $q$-oscillator representation. On the other hand, the eigenfunctions are constructed by using a representation of the deformed affine Hecke algebra in [10]. Thus our formula above may be regarded as a bridge between two approaches to studying integrable stochastic systems by means of the quantum affine algebra and the affine Hecke algebra.

The paper is organized as follows. In Section 2 we give the definition of the multi-species $q$-Boson system. In Section 3 the algebraic construction of the eigenfunctions is explained following [10]. In Section 4 we introduce the 
algebra of $q$-deformed bosons and its Fock representation, and discuss the integrability of the multi-species $q$-Boson system with periodic boundary. In Section 5 we state and prove the main theorem.

Throughout this paper we denote by $\prod_{i \in I}^{\curvearrowright} A_{i}$ or $\prod_{a \leq i \leq b}^{\curvearrowright} A_{i}$ the ordered product $A_{a} A_{a+1} \ldots A_{b-1} A_{b}$ of non-commutative elements $A_{i}$ labeled with integers in the interval $I=[a, b]$. Similarly we write $\prod_{i \in I}^{\curvearrowleft} A_{i}$ or $\prod_{a \leq i \leq b}^{\curvearrowleft} A_{i}$ for the reverse ordered product $A_{b} A_{b-1} \ldots A_{a+1} A_{a}$. We often abbreviate the sequence of positive integers $(a, \ldots, a)$ of length $m$ to $a^{m}$. For example, we write $(3,3,2,2,2,1)$ as $\left(3^{2}, 2^{3}, 1^{1}\right)$.

\section{The multi-species $q$-Boson system}

Hereafter we fix a real number $0<q<1$ and a positive integer $r$. The multi-species $q$-Boson system is a stochastic particle system on one-dimensional discrete space with continuous time. We consider the system on the infinite lattice $\boldsymbol{Z}$. The number of particles is finite and each particle is colored with a positive integer which is less than or equal to $r$. The particles can occupy the same site simultaneously. One particle may move to the left nearest-neighboring site independently for each site. The transition rate is given by

$$
\frac{1-q^{2 n_{a}}}{1-q^{2}} q^{2 \sum_{p=a+1}^{r} n_{p}},
$$

where $a$ is the color of the moving particle and $n_{p}(1 \leq p \leq r)$ is the number of particles with color $p$ in the cluster from which the moving particle leaves. For example the rate of the transition described in Figure 1 is equal to $\left(1-q^{2 \cdot 2}\right) q^{2(1+1)} /\left(1-q^{2}\right)$ because $a=2, n_{2}=2$ and $n_{3}=n_{4}=1$.

We denote by $k_{a}(1 \leq a \leq r)$ the number of particles with color $a$, and by $k=\sum_{a=1}^{r} k_{a}$ the total number of particles. They are conserved in the 


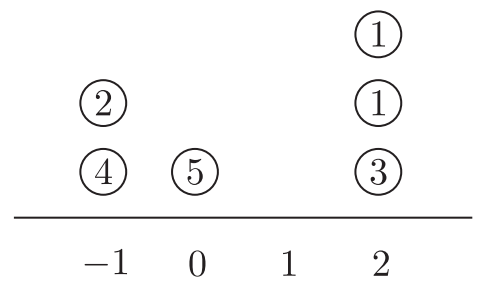

Fig. 2

process. We introduce two sets

$$
I_{k_{1}, \ldots, k_{r}}=\left\{\vec{v}=\left(v_{1}, \ldots, v_{k}\right) \in\{1, \ldots, r\}^{k} \mid \#\left\{j \mid v_{j}=a\right\}=k_{a}(1 \leq \forall a \leq r)\right\}
$$

and

$$
L_{k}^{+}=\left\{\vec{x}=\left(x_{1}, \ldots, x_{k}\right) \in \boldsymbol{Z}^{k} \mid x_{1} \geq \cdots \geq x_{k}\right\},
$$

and define

$$
\begin{gathered}
\mathscr{S}_{k_{1}, \ldots, k_{r}}=\left\{(\vec{x}, \vec{v}) \in L_{k}^{+} \times I_{k_{1}, \ldots, k_{r}} \mid \text { For any } 1 \leq i \leq k,\right. \\
\text { if } \left.x_{i}=x_{i+1} \text { then } v_{i} \leq v_{i+1} \cdot\right\} .
\end{gathered}
$$

We identify $\mathscr{S}_{k_{1}, \ldots, k_{r}}$ with the set of configurations of $k_{a}$ bosonic particles with color $a(1 \leq a \leq r)$ on $Z$ by assigning to $(\vec{x}, \vec{v})$ the configuration such that the particles with color $v_{1}, \ldots, v_{k}$ are on the sites $x_{1}, \ldots, x_{k}$, respectively. For example, the configuration in Figure 2 corresponds to the pair of $\vec{x}=(2,2,2,0$, $-1,-1)$ and $\vec{v}=(1,1,3,5,2,4)$.

Denote by $F\left(\mathscr{S}_{k_{1}, \ldots, k_{r}}\right)$ the set of $\boldsymbol{C}$-valued functions on $\mathscr{S}_{k_{1}, \ldots, k_{r}}$. The backward generator $\mathscr{H}$ of the multi-species $q$-Boson system is the linear operator on $F\left(\mathscr{S}_{k_{1}, \ldots, k_{r}}\right)$ defined by

$$
(\mathscr{H} h)(\vec{x}, \vec{v})=\sum_{\substack{(\vec{y}, \vec{\mu}) \in \mathscr{S}_{k_{1}, \ldots, k_{r}} \\(\vec{y}, \vec{\mu}) \neq(\vec{x}, \vec{v})}} q(\vec{x}, \vec{v} \mid \vec{y}, \vec{\mu})\{h(\vec{y}, \vec{\mu})-h(\vec{x}, \vec{v})\} \quad\left(h \in F\left(\mathscr{S}_{k_{1}, \ldots, k_{r}}\right)\right),
$$

where $q(\vec{x}, \vec{v} \mid \vec{y}, \vec{\mu})$ is the transition rate $(2.1)$ from $(\vec{x}, \vec{v})$ to $(\vec{y}, \vec{\mu})$.

\section{Algebraic construction of the eigenfunctions}

In this section we recall the construction of the eigenfunctions of the backward generator $\mathscr{H}$ following [10]. There, the eigenfunctions are expressed in terms of the root system and the Weyl group of type $A_{k-1}$. Instead, here we represent them in a combinatorial way. 
Let $U=\bigoplus_{a=1}^{r} C u_{a}$ be the $r$-dimensional vector space. We denote by $\left(U^{\otimes k}\right)_{k_{1}, \ldots, k_{r}}$ the subspace of $U^{\otimes k}$ spanned by the monomial vectors $u_{v_{1}} \otimes \cdots \otimes$ $u_{v_{k}}$ with $\left(v_{1}, \ldots, v_{k}\right) \in I_{k_{1}, \ldots, k_{r}}$. For a function $\gamma$ on $L_{k}^{+}$taking values in $\left(U^{\otimes k}\right)_{k_{1}, \ldots, k_{r}}$, we define $\boldsymbol{C}$-valued functions $\gamma_{\vec{v}}\left(\vec{v} \in I_{k_{1}, \ldots, k_{r}}\right)$ on $L_{k}^{+}$by the following relation:

$$
\gamma(\vec{x})=\sum_{\vec{v} \in I_{k_{1}, \ldots, k r}} q^{t(\vec{v})} \gamma_{\vec{v}}(\vec{x}) u_{v_{1}} \otimes \cdots \otimes u_{v_{k}} \quad\left(\vec{x} \in L_{k}^{+}\right),
$$

where $t(\vec{v})$ is the inversion number of $\vec{v}$ :

$$
t(\vec{v})=\#\left\{(i, j) \mid 1 \leq i<j \leq k \text { and } v_{i}>v_{j}\right\} .
$$

Denote by $\mathscr{F}\left(L_{k}^{+},\left(U^{\otimes k}\right)_{k_{1}, \ldots, k_{r}}\right)$ the space of functions $\gamma: L_{k}^{+} \rightarrow\left(U^{\otimes k}\right)_{k_{1}, \ldots, k_{r}}$ such that $\gamma_{\ldots, v_{i}, v_{i+1}, \ldots}=\gamma_{\ldots, v_{i+1}, v_{i}, \ldots}$ on the diagonal set $\left\{\vec{x} \in L_{k}^{+} \mid x_{i}=x_{i+1}\right\}$ for any $1 \leq i<k$. Then there exists an isomorphism $\varphi: F\left(\mathscr{S}_{k_{1}, \ldots, k_{r}}\right) \rightarrow \mathscr{F}\left(L_{k}^{+}\right.$, $\left.\left(U^{\otimes k}\right)_{k_{1}, \ldots, k_{r}}\right)$ whose inverse is given by

$$
\varphi^{-1}: \mathscr{F}\left(L_{k}^{+},\left(U^{\otimes k}\right)_{k_{1}, \ldots, k_{r}}\right) \rightarrow F\left(\mathscr{S}_{k_{1}, \ldots, k_{r}}\right), \quad\left(\varphi^{-1} \gamma\right)(\vec{x}, \vec{v})=\gamma_{\vec{v}}(\vec{x}) .
$$

We define the linear operator $R \in \operatorname{End}\left(U^{\otimes 2}\right)$ by

$$
R\left(u_{a} \otimes u_{b}\right)= \begin{cases}q u_{b} \otimes u_{a} & (a>b) \\ u_{a} \otimes u_{a} & (a=b) \\ \left(1-q^{2}\right) u_{a} \otimes u_{b}+q u_{b} \otimes u_{a} & (a<b) .\end{cases}
$$

For $1 \leq i<k$, we denote by $R_{i}$ the linear operator acting on the tensor product of the $i$-th and the $(i+1)$-th components of $U^{\otimes k}$ as the operator $R$. Then it holds that

$$
\begin{aligned}
& \left(R_{i}-1\right)\left(R_{i}+q^{2}\right)=0 \quad(1 \leq i<k), \\
& R_{i} R_{i+1} R_{i}=R_{i+1} R_{i} R_{i+1} \quad(1 \leq i \leq k-2), \\
& R_{i} R_{j}=R_{j} R_{i} \quad(|i-j| \geq 2) .
\end{aligned}
$$

Hence the operators $R_{i}(1 \leq i<k)$ define an action of the Hecke algebra of type $A_{k-1}$ [5]. Note that the subspace $\left(U^{\otimes k}\right)_{k_{1}, \ldots, k_{r}}$ is invariant under the action.

Hereafter we set

$$
f(z, w)=\frac{z-q^{2} w}{z-w}, \quad g(z, w)=-\frac{\left(1-q^{2}\right) z}{z-w}=f(w, z)-1 .
$$

For $1 \leq i<k$ and $z, w \in C$, set

$$
Y_{i}(z, w)=\frac{1}{f(z, w)}\left(R_{i}+g(z, w)\right) \in \operatorname{End}\left(U^{\otimes k}\right) .
$$


For $\tau \in \Im_{k}$ and $\vec{z}=\left(z_{1}, \ldots, z_{k}\right) \in \boldsymbol{C}^{k}$, the linear operator $\phi(\tau ; \vec{z})$ acting on $U^{\otimes k}$ is uniquely determined from the following properties:

$$
\phi(1 ; \vec{z})=1, \quad \phi\left(\sigma_{i} \tau ; \vec{z}\right)=Y_{i}\left(z_{\tau^{-1}(i)}, z_{\tau^{-1}(i+1)}\right) \phi(\tau ; \vec{z}),
$$

where $\sigma_{i}=(i, i+1)(1 \leq i<k)$ is the transposition.

Lemma 3.1. For $\tau, \tau^{\prime} \in \mathfrak{S}_{k}$ and $\vec{z}=\left(z_{1}, \ldots, z_{k}\right) \in \boldsymbol{C}^{k}$, it holds that

$$
\phi\left(\tau^{\prime} \tau ; \vec{z}\right)=\phi\left(\tau^{\prime} ; z_{\tau^{-1}(1)}, \ldots, z_{\tau^{-1}(k)}\right) \phi(\tau ; \vec{z}) .
$$

Proof. It follows from the properties which define the operator $\phi(\tau ; \vec{z})$.

Now we write down the formula of the eigenfunctions.

Definition 3.2. For $\vec{\mu}=\left(\mu_{1}, \ldots, \mu_{k}\right) \in I_{k_{1}, \ldots, k_{r}}$ and $\vec{z}=\left(z_{1}, \ldots, z_{k}\right) \in \boldsymbol{C}^{k}$, we define the function $h_{\vec{z}}^{\vec{\mu}}$ on $L_{k}^{+}$taking values in $U^{\otimes k}$ by

$$
h_{\vec{z}}^{\vec{\mu}}(\vec{x})=\prod_{1 \leq i<j \leq k} f\left(z_{i}, z_{j}\right) \sum_{\tau \in \mathbb{S}_{k}} \prod_{i=1}^{k}\left(\frac{z_{\tau^{-1}(i)}}{1+z_{\tau^{-1}(i)}}\right)^{x_{i}} \phi(\tau ; \vec{z})\left(u_{\mu_{1}} \otimes \cdots \otimes u_{\mu_{k}}\right) .
$$

Theorem 3.3 [10]. The function $h_{\vec{z}}^{\vec{\mu}}$ belongs to $\mathscr{F}\left(L_{k}^{+},\left(U^{\otimes k}\right)_{k_{1}, \ldots, k_{r}}\right)$ and $\varphi^{-1} h_{\vec{z}}^{\vec{\mu}}$ is an eigenfunction of the backward generator $\mathscr{H}$ with the eigenvalue $\sum_{i=1}^{k} z_{i}^{-1}$.

Remark 3.4. In [10] the eigenfunctions are parametrized by $m \in U^{\otimes k}$ and $\lambda \in\left(\oplus_{i=1}^{k} \boldsymbol{R} v_{i}\right)^{*}$. They correspond to $\vec{\mu}$ and $\vec{z}$ respectively via the relations $m=u_{\mu_{1}} \otimes \cdots \otimes u_{\mu_{k}}$ and $e^{\lambda\left(v_{i}\right)}=z_{i} /\left(1+z_{i}\right)$. We also put the additional factor $\prod_{1 \leq i<j \leq k} f\left(z_{i}, z_{j}\right)$ to make the function $h_{\vec{z}}^{\vec{\mu}}$ have symmetry (see Proposition 5.10 below).

Remark 3.5. When $r=1$, the vector space $U^{\otimes k}$ is one-dimensional and it holds that

$$
h_{\vec{z}}^{1^{k}}(\vec{x})=\left(\sum_{\sigma \in \mathfrak{S}_{k}} \prod_{1 \leq i<j \leq k} \frac{z_{\sigma(i)}-q^{2} z_{\sigma(j)}}{z_{\sigma(i)}-z_{\sigma(j)}} \prod_{i=1}^{k}\left(\frac{z_{\sigma(i)}}{1+z_{\sigma(i)}}\right)^{x_{i}}\right)\left(u_{1} \otimes \cdots \otimes u_{1}\right) .
$$

Thus we recover the eigenfunctions for the $q$-Boson system constructed in [2] by changing the parameters $z_{i} \mapsto-z_{i}^{-1}$.

\section{The algebra of $q$-deformed bosons and integrability}

\subsection{The $L$-operator}

We define the algebra $\mathscr{B}$ of multi-component $q$-deformed bosons to be the unital associative $\boldsymbol{C}$-algebra with the generators $\beta_{a}, \beta_{a}^{*}, q^{ \pm N_{a}}(1 \leq a \leq r)$ 
satisfying

$$
\begin{array}{ll}
q^{N_{a}} \beta_{a}=q^{-1} \beta_{a} q^{N_{a}}, & q^{N_{a}} \beta_{a}^{*}=q \beta_{a}^{*} q^{N_{a}}, \\
\beta_{a} \beta_{a}^{*}=1-q^{2} q^{2 N_{a}}, & \beta_{a}^{*} \beta_{a}=1-q^{2 N_{a}}
\end{array}
$$

and such that the elements $\beta_{a}, \beta_{a}^{*}, q^{ \pm N_{a}}$ and $\beta_{b}, \beta_{b}^{*}, q^{ \pm N_{b}}$ commute unless $a=b$. We abbreviate $\prod_{a=1}^{r}\left(q^{N_{a}}\right)^{n_{a}}$ to $q^{\sum_{a=1}^{r} n_{a} N_{a}}$ for $n_{1}, \ldots, n_{r} \in \boldsymbol{Z}$.

In [4] a generalized $L$-operator of higher rank is introduced. We make use of it with parameters fixed (see Remark 4.2 below). The $L$-operator $L(z)=$ $\left(L(z)_{a b}\right)_{a, b=0}^{r} \in \mathscr{B} \otimes \operatorname{End}\left(C^{r+1}\right)$ is defined by

$$
\begin{aligned}
& L(z)_{00}=1+z q^{2 \sum_{p=1}^{r} N_{p},} \\
& L(z)_{0 a}=\beta_{a}^{*}, \quad L(z)_{a 0}=z \beta_{a} q^{2 \sum_{p=a+1}^{r} N_{p}} \quad(1 \leq a \leq r), \\
& L(z)_{a b}= \begin{cases}0 & (1 \leq a<b \leq r) \\
z q^{2 \sum_{p=a+1}^{r} N_{p}} & (1 \leq a=b \leq r) \\
z \beta_{a} \beta_{b}^{*} q^{2 \sum_{p=a+1}^{r} N_{p}} & (1 \leq b<a \leq r) .\end{cases}
\end{aligned}
$$

We denote the matrix unit by $E_{a b}=\left(\delta_{a i} \delta_{j b}\right)_{i, j=0}^{r}$ and define $L(z) \otimes L(w) \in$ $\mathscr{B} \otimes \operatorname{End}\left(\boldsymbol{C}^{r+1} \otimes \boldsymbol{C}^{r+1}\right)$ by

$$
L(z) \otimes L(w)=\sum_{a, b=0}^{r} \sum_{c, d=0}^{r}\left(L(z)_{a b} L(w)_{c d}\right) \otimes E_{a b} \otimes E_{c d} .
$$

Then the following relation holds.

Proposition 4.1 [4]. The L-operator satisfies the Yang-Baxter equation

$$
\check{R}(z / w)[L(z) \otimes L(w)]=[L(w) \otimes L(z)] \check{R}(z / w),
$$

where $\check{R}(z) \in \operatorname{End}\left(\boldsymbol{C}^{r+1} \otimes \boldsymbol{C}^{r+1}\right)$ is given by

$$
\begin{aligned}
\check{R}(z)= & \left(z-q^{2}\right) \sum_{a=1}^{r} E_{a a} \otimes E_{a a}+\left(1-q^{2}\right) \sum_{1 \leq a<b \leq r}\left(z E_{a a} \otimes E_{b b}+E_{b b} \otimes E_{a a}\right) \\
& +(z-1) \sum_{1 \leq a<b \leq r}\left(E_{a b} \otimes E_{b a}+q^{2} E_{b a} \otimes E_{a b}\right) .
\end{aligned}
$$

Remark 4.2. Our $L$-operator $L(z)$ is obtained from that defined in [4] by setting $t=q^{-2}, \phi_{a}=\beta_{a}^{*}, \phi_{a}^{\dagger}=\beta_{a}, k_{a}=q^{2 N_{a}}, u=-1$ and $v=0$.

\subsection{The monodromy matrix}

We introduce commutative copies $\mathscr{B}^{(i)}(i \in \boldsymbol{Z})$ of the algebra $\mathscr{B}$. Denote the generators of $\mathscr{B}^{(i)}$ by $\beta_{a, i}, \beta_{a, i}^{*}$ and $q^{ \pm N_{a, i}}(1 \leq a \leq r)$. The elements $\beta_{a, i}$, 
$\beta_{a, i}^{*}, q^{ \pm N_{a, i}}$ satisfy the relations (4.1) and commute with $\beta_{b, j}, \beta_{b, j}^{*}, q^{ \pm N_{b, j}}$ such that $(a, i) \neq(b, j)$

We assign $\mathscr{B}^{(i)}$ to the $i$-th site on the one-dimensional lattice $\boldsymbol{Z}$, and define the $L$-operator $L^{(i)}(z)$ by $(4.2)$ with $\beta_{a}, \beta_{a}^{*}, q^{ \pm N_{a}}$ replaced by $\beta_{a, i}, \beta_{a, i}^{*}, q^{ \pm N_{a, i}}$ $(1 \leq a \leq r)$.

Let $M$ and $M^{\prime}$ be integers such that $M^{\prime} \leq M$. Denote by $\mathscr{B}^{\left[M^{\prime}, M\right]}$ the algebra generated by $\mathscr{B}^{(i)}\left(M^{\prime} \leq i \leq M\right)$. We define the monodromy matrix $\boldsymbol{T}^{\left[M^{\prime}, M\right]}(z)$ by

$$
\boldsymbol{T}^{\left[M^{\prime}, M\right]}(z)=L^{\left(M^{\prime}\right)}(z) L^{\left(M^{\prime}+1\right)}(z) \ldots L^{(M-1)}(z) L^{(M)}(z),
$$

which belongs to $\mathscr{B}^{\left[M^{\prime}, M\right]} \otimes \operatorname{End}\left(\boldsymbol{C}^{r+1}\right)$. Denote its matrix element by $\boldsymbol{T}^{\left[M^{\prime}, M\right]}(z)_{a b}(0 \leq a, b \leq r)$. From the Yang-Baxter equation (4.3) we have

$$
\check{R}(z / w)\left[\boldsymbol{T}^{\left[M^{\prime}, M\right]}(z) \otimes \boldsymbol{T}^{\left[M^{\prime}, M\right]}(w)\right]=\left[\boldsymbol{T}^{\left[M^{\prime}, M\right]}(w) \otimes \boldsymbol{T}^{\left[M^{\prime}, M\right]}(z)\right] \check{R}(z / w),
$$

where $\boldsymbol{T}^{\left[M^{\prime}, M\right]}(z) \otimes \boldsymbol{T}^{\left[M^{\prime}, M\right]}(w) \in \mathscr{B}^{\left[M^{\prime}, M\right]} \otimes \operatorname{End}\left(\boldsymbol{C}^{r+1} \otimes \boldsymbol{C}^{r+1}\right)$ is defined by

$$
\boldsymbol{T}^{\left[M^{\prime}, M\right]}(z) \otimes \boldsymbol{T}^{\left[M^{\prime}, M\right]}(w)=\sum_{a, b=0}^{r} \sum_{c, d=0}^{r}\left(\boldsymbol{T}^{\left[M^{\prime}, M\right]}(z)_{a b} \boldsymbol{T}^{\left[M^{\prime}, M\right]}(w)_{c d}\right) \otimes E_{a b} \otimes E_{c d}
$$

Now set

$$
A^{\left[M^{\prime}, M\right]}(z)=\boldsymbol{T}^{\left[M^{\prime}, M\right]}(z)_{00}, \quad C_{a}^{\left[M^{\prime}, M\right]}(z)=\boldsymbol{T}^{\left[M^{\prime}, M\right]}(z)_{a 0} \quad(1 \leq a \leq r) .
$$

The equality (4.4) implies the following commutation relations:

Proposition 4.3. We fix $M^{\prime}$ and $M$, and abbreviate $X^{\left[M^{\prime}, M\right]}(z)\left(X=A, C_{a}\right)$ to $X(z)$. It holds that

$$
\begin{aligned}
& A(z) A(w)=A(w) A(z), \quad C_{a}(z) C_{a}(w)=C_{a}(w) C_{a}(z) \quad(1 \leq a \leq r), \\
& C_{a}(z) A(w)=f(z, w) A(w) C_{a}(z)+g(z, w) A(z) C_{a}(w) \quad(1 \leq a \leq r), \\
& q^{2} C_{b}(z) C_{a}(w)=f(z, w) C_{a}(w) C_{b}(z) \\
& -g(w, z) C_{a}(z) C_{b}(w) \quad(1 \leq b<a \leq r),
\end{aligned}
$$

where $f(z, w)$ and $g(z, w)$ are defined by (3.3).

Moreover the following recurrence relations hold.

Proposition 4.4. Suppose that $M \geq 2$. Then it holds that

$$
A^{[1, M]}(z)=\left(1+z q^{2 \sum_{p=1}^{r} N_{p, 1}}\right) A^{[2, M]}(z)+\sum_{p=1}^{r} \beta_{p, 1}^{*} C_{p}^{[2, M]}(z)
$$




$$
\begin{aligned}
C_{a}^{[1, M]}(z)= & z q^{2 \sum_{p=a+1}^{r} N_{p, 1}}\left\{\beta_{a, 1}\left(A^{[2, M]}(z)+\sum_{p=1}^{a-1} \beta_{p, 1}^{*} C_{p}^{[2, M]}(z)\right)\right. \\
& \left.+C_{a}^{[2, M]}(z)\right\}, \\
C_{a}^{[1, M]}(z)= & C_{a}^{[1, M-1]}(z)\left(1+z q^{\left.2 \sum_{p=1}^{r} N_{p, M}\right)}\right. \\
& +z \sum_{b=1}^{r} \boldsymbol{T}_{a, b}^{[1, M-1]}(z) \beta_{b, M} q^{2 \sum_{p=b+1}^{r} N_{p, M}}
\end{aligned}
$$

for $1 \leq a \leq r$.

Proof. Write down the matrix elements in the zeroth column of

$$
\boldsymbol{T}^{[1, M]}(z)=L^{(1)}(z) \boldsymbol{T}^{[2, M]}(z)=\boldsymbol{T}^{[1, M-1]}(z) L^{(M)}(z),
$$

and we get the relations above.

\subsection{The integrability of the multi-species $q$-Boson model with periodic boundary}

The Fock representation $\mathscr{F}$ of the algebra $\mathscr{B}$ is the vector space

$$
\mathscr{F}=\bigoplus_{m_{1}, \ldots, m_{r} \in \boldsymbol{Z}_{\geq 0}} \boldsymbol{C}\left|m_{1}, \ldots, m_{r}\right\rangle
$$

equipped with the left $\mathscr{B}$-module structure defined by

$$
\begin{aligned}
& q^{N_{a}}\left|m_{1}, \ldots, m_{r}\right\rangle=q^{m_{a}}\left|m_{1}, \ldots, m_{r}\right\rangle, \\
& \beta_{a}^{*}\left|m_{1}, \ldots, m_{r}\right\rangle=\left|m_{1}, \ldots, m_{a}+1, \ldots, m_{r}\right\rangle, \\
& \beta_{a}\left|m_{1}, \ldots, m_{r}\right\rangle=\left(1-q^{2 m_{a}}\right)\left|m_{1}, \ldots, m_{a}-1, \ldots, m_{r}\right\rangle .
\end{aligned}
$$

The right action on its dual module

$$
\mathscr{F}^{*}=\bigoplus_{m_{1}, \ldots, m_{r} \in \boldsymbol{Z}_{\geq 0}} \boldsymbol{C}\left\langle m_{1}, \ldots, m_{r}\right|
$$

is given by

$$
\begin{aligned}
& \left\langle m_{1}, \ldots, m_{r}\right| q^{N_{a}}=q^{m_{a}}\left\langle m_{1}, \ldots, m_{r}\right|, \\
& \left\langle m_{1}, \ldots, m_{r}\right| \beta_{a}=\left(1-q^{2\left(m_{a}+1\right)}\right)\left\langle m_{1}, \ldots, m_{a}+1, \ldots, m_{r}\right|, \\
& \left\langle m_{1}, \ldots, m_{r}\right| \beta_{a}^{*}= \begin{cases}0 & \left(m_{a}=0\right) \\
\left\langle m_{1}, \ldots, m_{a}-1, \ldots, m_{r}\right| & \left(m_{a} \geq 1\right) .\end{cases}
\end{aligned}
$$


Let $M$ be a positive integer. We introduce $M$ copies $\mathscr{F}^{(i)}(1 \leq i \leq M)$ of the vector space $\mathscr{F}$ and set $\mathscr{F}^{[1, M]}=\mathscr{F}^{(1)} \otimes \cdots \otimes \mathscr{F}^{(M)}$. The algebra $\mathscr{B}^{[1, M]}$ acts on $\mathscr{F}^{[1, M]}$ in such a way that $\mathscr{B}^{(i)}$ acts on the $i$-th component $\mathscr{F}^{(i)}$ as given by (4.10). Then we define the transfer matrix $\tau(z) \in \operatorname{End}\left(\mathscr{F}^{[1, M]}\right)$ by

$$
\tau(z)=\operatorname{tr}_{C^{r+1}}\left(\boldsymbol{T}^{[1, M]}(z)\right)=\sum_{a=0}^{r} \boldsymbol{T}^{[1, M]}(z)_{a a} .
$$

Note that $\tau(z)$ is a polynomial in $z$ of degree $M$. Now define the operators $H_{n}(1 \leq n \leq M)$ by the expansion $\tau(z)=\sum_{n=0}^{M} H_{n} z^{n}$. The relation (4.4) implies that

$$
\check{R}(z / w)\left[\boldsymbol{T}^{[1, M]}(z) \otimes \boldsymbol{T}^{[1, M]}(w)\right] \check{R}(z / w)^{-1}=\boldsymbol{T}^{[1, M]}(w) \otimes \boldsymbol{T}^{[1, M]}(z) .
$$

Taking the trace over $\boldsymbol{C}^{r+1} \otimes C^{r+1}$, we get

$$
\tau(z) \tau(w)=\tau(w) \tau(z) .
$$

Hence $H_{n}(0 \leq n \leq M)$ commutes with each other.

By direct calculation we see that $H_{0}=1$ and

$$
H_{1}=M+\sum_{i=1}^{M} h_{i-1, i}, \quad h_{i-1, i}=\sum_{a=1}^{r}\left(\beta_{a, i-1}^{*}-\beta_{a, i}^{*}\right) \beta_{a, i} q^{2 \sum_{p=a+1}^{r} N_{a, i}},
$$

where the index $i$ should read modulo $M$. Note that

$$
\begin{aligned}
h_{12}\left(\left|m_{1}, \ldots, m_{r}\right\rangle \otimes\left|n_{1}, \ldots, n_{r}\right\rangle\right) \\
=\sum_{a=1}^{r} q^{2 \sum_{p=a+1}^{r} n_{p}}\left(1-q^{2 n_{a}}\right) \\
\quad \times\left(\left|\ldots, m_{a}+1, \ldots\right\rangle \otimes\left|\ldots, n_{a}-1, \ldots\right\rangle-\left|\ldots, m_{a}, \ldots\right\rangle \otimes\left|\ldots, n_{a}, \ldots\right\rangle\right) .
\end{aligned}
$$

Let $\mathscr{S}_{M}^{\text {per }}$ be the set of configurations of bosonic particles with $r$ species on the one-dimensional lattice of length $M$ with periodic boundary. We assign each configuration $\mathbf{s}$ to the vector $|\mathbf{s}\rangle=\bigotimes_{i=1}^{M}\left(\left|n_{1}^{(i)}, \ldots, n_{r}^{(i)}\right\rangle\right)$ of $\mathscr{F}^{[1, M]}$, where $n_{a}^{(i)}$ is the number of particles with color $a$ at the $i$-th site in the configuration s. Then we see that

$$
\left(H_{1}-M\right)|\mathbf{s}\rangle=\left(1-q^{2}\right) \sum_{\mathbf{s}^{\prime} \in \mathscr{S}_{M}^{\text {per }} \backslash\{\mathbf{s}\}} q\left(\mathbf{s} \mid \mathbf{s}^{\prime}\right)\left(\left|\mathbf{s}^{\prime}\right\rangle-|\mathbf{s}\rangle\right),
$$

where $q\left(\mathbf{s} \mid \mathbf{s}^{\prime}\right)$ is the transition rate of the multi-species $q$-Boson system from $\mathbf{s}$ to $\mathbf{s}^{\prime}$ given by (2.1). Therefore $H_{1}$ gives the transition rate matrix of the 
system with periodic boundary. The operators $H_{n}(2 \leq n \leq M)$ generated from the transfer matrix commute with it. Thus we may regard the multispecies $q$-Boson system as integrable.

\section{Main theorem and its proof}

\subsection{Main theorem}

We now turn back to the system on the infinite lattice $\boldsymbol{Z}$. We introduce countably many copies $\mathscr{B}^{(i)}$ and $\mathscr{F}^{(i)}(i \in \boldsymbol{Z})$ of the algebra $\mathscr{B}$ and its Fock representation $\mathscr{F}$, respectively, and assign $\mathscr{B}^{(i)}$ and $\mathscr{F}^{(i)}$ to the $i$-th site of the lattice $Z$. For two integers $M^{\prime}$ and $M$ such that $M^{\prime} \leq M$, we denote by $\mathscr{B}^{\left[M^{\prime}, M\right]}$ the algebra generated by $\mathscr{B}^{(i)}\left(M^{\prime} \leq i \leq M\right)$ and set $\mathscr{F}^{\left[M^{\prime}, M\right]}=$ $\mathscr{F}^{\left(M^{\prime}\right)} \otimes \mathscr{F}^{\left(M^{\prime}+1\right)} \otimes \cdots \otimes \mathscr{F}^{(M)}$. Set $|\mathbf{0}\rangle=|0, \ldots, 0\rangle \in \mathscr{F}$ and $\langle\mathbf{0}|=\langle 0, \ldots, 0|$ $\in \mathscr{F}^{*}$. We denote the vacuum vector and its dual on the interval $\left[M^{\prime}, M\right]$ by

$$
\begin{aligned}
|\operatorname{vac}\rangle_{\left[M^{\prime}, M\right]} & =|\mathbf{0}\rangle \otimes|\mathbf{0}\rangle \otimes \cdots \otimes|\mathbf{0}\rangle \in \mathscr{F}^{\left[M^{\prime}, M\right]}, \\
{\left[M^{\prime}, M\right] } & \langle\mathrm{vac}|=\langle\mathbf{0}| \otimes\langle\mathbf{0}| \otimes \cdots \otimes\langle\mathbf{0}| \in\left(\mathscr{F}^{*}\right)^{\left[M^{\prime}, M\right]}
\end{aligned}
$$

For simplicity we write

$$
\langle X\rangle_{\left[M^{\prime}, M\right]}={ }_{\left[M^{\prime}, M\right]}\langle\operatorname{vac}|X| \operatorname{vac}\rangle_{\left[M^{\prime}, M\right]} \quad\left(X \in \mathscr{B}^{\left[M^{\prime}, M\right]}\right) .
$$

Proposition 5.1. Suppose that $\left(x_{1}, \ldots, x_{k}\right) \in L_{k}^{+}$and $\left(\mu_{1}, \ldots, \mu_{k}\right)$, $\left(v_{1}, \ldots, v_{k}\right) \in I_{k_{1}, \ldots, k_{r}}$. Then the following quantity does not depend on the choice of $M$ and $M^{\prime}$ such that $M \geq x_{1}$ and $x_{k} \geq M^{\prime}$ :

$$
\prod_{i=1}^{k} \frac{z_{i}^{M^{\prime}-1}}{\left(1+z_{i}\right)^{M}}\left\langle\prod_{1 \leq i \leq k}^{\curvearrowright} C_{\mu_{i}}^{\left[M, M^{\prime}\right]}\left(z_{i}\right) \prod_{1 \leq i \leq k} \beta_{v_{i}, x_{i}}^{*}\right\rangle_{\left[M^{\prime}, M\right]} .
$$

Proof. Let $M \geq 2$ and $X \in \mathscr{B}^{[1, M]}$. From Proposition 4.4 it holds that

$$
\begin{aligned}
& C_{a}^{[1, M+1]}(z) X|\mathrm{vac}\rangle_{[1, M+1]}=(1+z) C_{a}^{[1, M]}(z) X|\mathrm{vac}\rangle_{[1, M]}, \\
& C_{a}^{[0, M]}(z) X|\mathrm{vac}\rangle_{[0, M]}=z C_{a}^{[1, M]}(z) X|\mathrm{vac}\rangle_{[1, M]} .
\end{aligned}
$$

for $1 \leq a \leq r$. The properties above imply that the value (5.1) is equal to that with $M^{\prime}$ and $M$ replaced by $x_{k}$ and $x_{1}$, respectively.

Now we introduce the following function.

Definition 5.2. For $\vec{\mu}=\left(\mu_{1}, \ldots, \mu_{k}\right) \in I_{k_{1}, \ldots, k_{r}}$ and $\vec{z}=\left(z_{1}, \ldots, z_{k}\right) \in \boldsymbol{C}^{k}$, we define the function $\psi_{\vec{z}}^{\vec{\mu}}$ on $L_{k}^{+}$taking values in $\left(U^{\otimes k}\right)_{k_{1}, \ldots, k_{r}}$ by 


$$
\begin{aligned}
\psi_{\vec{z}}^{\vec{\mu}}(\vec{x})= & \left(1-q^{2}\right)^{-k} \sum_{\vec{v} \in I_{k_{1}, \ldots, k_{r}}} \prod_{i=1}^{k} \frac{z_{i}^{M^{\prime}-1}}{\left(1+z_{i}\right)^{M}}\left\langle\prod_{1 \leq i \leq k}^{\curvearrowright} C_{\mu_{i}}^{\left[M^{\prime}, M\right]}\left(z_{i}\right) \prod_{1 \leq i \leq k} \beta_{v_{i}, x_{i}}^{*}\right\rangle_{\left[M^{\prime}, M\right]} \\
& \times q^{t(\vec{v})-t(\vec{\mu})} u_{v_{1}} \otimes \cdots \otimes u_{v_{k}}
\end{aligned}
$$

where $M$ and $M^{\prime}$ are integers such that $M \geq x_{1}$ and $x_{k} \geq M^{\prime}$, and $t(\cdot)$ is the inversion number (3.1).

Example 5.3. Let us consider the case of $r=1$. In the following we drop the subscript 1 on $\beta_{1, i}, \beta_{1, i}^{*}, q^{N_{1, i}}$ and $C_{1}^{\left[M^{\prime}, M\right]}(z)$ for brevity. The $L$-operator is in the form of $2 \times 2$ matrix:

$$
L^{(i)}(z)=\left(\begin{array}{cc}
1+z q^{2 N_{i}} & \beta_{i}^{*} \\
z \beta_{i} & z
\end{array}\right)
$$

Then the operator $C^{\left[M^{\prime}, M\right]}(z)$ is defined by

$$
C^{\left[M^{\prime}, M\right]}(z)=\left(\begin{array}{ll}
0 & 1
\end{array}\right) L^{\left(M^{\prime}\right)}(z) L^{\left(M^{\prime}+1\right)}(z) \ldots L^{(M)}(z)\left(\begin{array}{l}
1 \\
0
\end{array}\right) .
$$

The function $\psi_{\vec{z}}^{\vec{\mu}}$ is defined only for $\vec{\mu}=(1, \ldots, 1)$ and we see that

$$
\begin{aligned}
\psi_{\vec{z}}^{1^{k}}(\vec{x})= & \left(1-q^{2}\right)^{-k} \prod_{i=1}^{k} \frac{z_{i}^{M^{\prime}-1}}{\left(1+z_{i}\right)^{M}}\left\langle\prod_{1 \leq i \leq k} C^{\left[M^{\prime}, M\right]}\left(z_{i}\right) \prod_{1 \leq i \leq k} \beta_{x_{i}}^{*}\right\rangle_{\left[M^{\prime}, M\right]} \\
& \times\left(u_{1} \otimes \cdots \otimes u_{1}\right) .
\end{aligned}
$$

Note that $\left[C^{\left[M^{\prime}, M\right]}(z), C^{\left[M^{\prime}, M\right]}(w)\right]=0$ because of Proposition 4.3. Hence the function $\psi_{\vec{z}}^{1^{k}}$ is symmetric in $\vec{z}=\left(z_{1}, \ldots, z_{k}\right)$.

Our main theorem is as follows.

Theorem 5.4. It holds that $h_{\vec{z}}^{\vec{\mu}}=\psi_{\vec{z}}^{\vec{\mu}}$ for any $\vec{\mu} \in I_{k_{1}, \ldots, k_{r}}$ and $\vec{z} \in C^{k}$.

We prove the theorem in the following subsections. Here we note that it gives us an expression of the eigenfunctions for the multi-species $q$-Boson system in terms of the $q$-deformed bosonic operators:

Corollary 5.5. For $\vec{\mu}=\left(\mu_{1}, \ldots, \mu_{k}\right) \in I_{k_{1}, \ldots, k_{r}}$ and $\vec{z}=\left(z_{1}, \ldots, z_{k}\right) \in \boldsymbol{C}^{k}$, we define the function $E_{\vec{z}}^{\vec{\mu}}$ on $\mathscr{S}_{k_{1}, \ldots, k_{r}}$ by

$$
E_{\vec{z}}^{\vec{\mu}}(\vec{x}, \vec{v})=\lim _{\substack{M \rightarrow \infty \\ M^{\prime} \rightarrow-\infty}} \prod_{i=1}^{k} \frac{z_{i}^{M^{\prime}-1}}{\left(1+z_{i}\right)^{M}}\left\langle\prod_{1 \leq i \leq k}^{\curvearrowright} C_{\mu_{i}}^{\left[M^{\prime}, M\right]}\left(z_{i}\right) \prod_{1 \leq i \leq k} \beta_{v_{i}, x_{i}}^{*}\right\rangle_{\left[M^{\prime}, M\right]} .
$$

Then $E_{\vec{z}}^{\vec{\mu}}$ is an eigenfunction of the backward generator $\mathscr{H}$ with the eigenvalue $\sum_{i=1}^{k} z_{i}^{-1}$. 
Proof. It follows from Theorem 3.3, Theorem 5.4 and the equality $E_{\vec{z}}^{\vec{\mu}}=$ $\left(1-q^{2}\right)^{k} q^{t(\vec{\mu})}\left(\varphi^{-1} \psi_{\vec{z}}^{\vec{\mu}}\right)$, where $\varphi^{-1}$ is the isomorphism defined by (3.2).

Remark 5.6. We proved above that $E_{\vec{z}}^{\vec{\mu}}$ is an eigenfunction by showing that it is equal to $\varphi^{-1} h_{\vec{z}}^{\vec{\mu}}$ up to a constant factor. The statement might be proved directly from the expression (5.4). Here we do not discuss this point further.

Remark 5.7. If $r=1$, then the equality $h_{\vec{z}}^{\vec{\mu}}=\psi_{\vec{z}}^{\vec{\mu}}$ in Theorem 5.4 can be derived from the combinatorial formula for a family of symmetric rational functions due to Borodin [1] as follows.

Note that $h_{\vec{z}}^{\vec{\mu}}$ and $\psi_{\vec{z}}^{\vec{\mu}}$ are defined only for $\vec{\mu}=1^{k}$ if $r=1$. Hence it suffices to prove the following equality for any $\vec{z} \in C^{k}$ and $\vec{x} \in L_{k}^{+}$:

$$
\begin{aligned}
& \sum_{\sigma \in \mathfrak{S}_{k}} \prod_{1 \leq i<j \leq k} \frac{z_{\sigma(i)}-q^{2} z_{\sigma(j)}}{z_{\sigma(i)}-z_{\sigma(j)}} \prod_{i=1}^{k}\left(\frac{z_{\sigma(i)}}{1+z_{\sigma(i)}}\right)^{x_{i}} \\
& \quad=\left(1-q^{2}\right)^{-k} \prod_{i=1}^{k}\left(z_{i}^{M^{\prime}-1}\left(1+z_{i}\right)^{-M}\right)\left\langle\prod_{1 \leq i \leq k} C^{\left[M^{\prime}, M\right]}\left(z_{i}\right) \prod_{1 \leq i \leq k} \beta_{x_{i}}^{*}\right\rangle_{\left[M^{\prime}, M\right]}
\end{aligned}
$$

(see Remark 3.5 and Example 5.3). Moreover we can assume that $x_{k} \geq 0$ and $M^{\prime}=0$ because of Proposition 5.9 which will be proved in the next subsection.

Following [1] we introduce the function (here we replaced the parameter $q$ in [1] with $q^{2}$ )

$$
F_{\vec{x}}\left(u_{1}, \ldots, u_{k}\right)=\frac{\left(1-q^{2}\right)^{k}}{\prod_{i=1}^{k}\left(1-s u_{i}\right)} \sum_{\sigma \in \mathscr{F}_{k}} \prod_{1 \leq i<j \leq k} \frac{u_{\sigma(i)}-q^{2} u_{\sigma(j)}}{u_{\sigma(i)}-u_{\sigma(j)}} \prod_{i=1}^{k}\left(\frac{u_{i}-s}{1-s u_{i}}\right)^{x_{i}}
$$

for $\vec{x} \in L_{k}^{+}$such that $x_{k} \geq 0$, where $s$ is a parameter. The combinatorial formula proved in [1] represents the function $F_{\vec{x}}$ as a partition function for path ensembles in the square grid. It can be rewritten in terms of the $q$-deformed bosons as follows.

Define the operator $\tilde{L}(u ; s)=\left(\tilde{L}(u ; s)_{a b}\right)_{a, b=0}^{1} \in \mathscr{B} \otimes \operatorname{End}\left(C^{2}\right)$ by

$$
\tilde{L}(u ; s)=\frac{1}{1-s u}\left(\begin{array}{cc}
1-s u q^{2 N} & u \beta^{*}\left(1-s^{2} q^{2 N}\right) \\
\beta & u-s q^{2 N}
\end{array}\right)
$$

and $\tilde{L}^{(i)}(u ; s)(i \in \boldsymbol{Z})$ in the same way as before. Then the vertex weight $w_{u}\left(i_{1}, j_{i} ; i_{2}, j_{2}\right)$ defined in [1] is realized as $w_{u}\left(i_{1}, j_{1} ; i_{2}, j_{2}\right)=\left\langle i_{1}\left|\tilde{L}(u ; s)_{j_{1} j_{2}}\right| i_{2}\right\rangle$ for $i_{1}, i_{2} \in \boldsymbol{Z}_{\geq 0}$ and $j_{1}, j_{2} \in\{0,1\}$. This correspondence implies that the combinatorial formula proved in [1] is rewritten as

$$
F_{\vec{x}}\left(u_{1}, \ldots, u_{k}\right)=\left\langle\prod_{1 \leq i \leq k} \tilde{C}^{[0, M]}\left(u_{i} ; s\right) \prod_{1 \leq i \leq k} \beta_{x_{i}}^{*}\right\rangle_{[0, M]}
$$


where $M$ is an arbitrary integer such that $M \geq x_{1}$ and $\tilde{C}^{[0, M]}(u ; s)$ is the operator acting on $\mathscr{F}^{[0, M]}$ defined by the right hand side of (5.3) with $M^{\prime}=0$ and $L^{(i)}(z)$ replaced by $\tilde{L}^{(i)}(u ; s)(0 \leq i \leq M)$.

Now we introduce the $s$-deformed $L$-operator

$$
L^{(i)}(z ; s)=\left(\begin{array}{cc}
1+z q^{2 N_{i}} & \beta_{i}^{*}\left(1-s^{2} q^{2 N_{i}}\right) \\
z \beta_{i} & z+s^{2} q^{2 N_{i}}
\end{array}\right) \quad(0 \leq i \leq M) .
$$

Note that it is equal to the $L$-operator (5.2) at $s=0$. For a parameter $\alpha \in C$ we define the linear operator $K(\alpha)$ acting on the Fock space $\mathscr{F}$ by $K(\alpha)|m\rangle=$ $\alpha^{m}|m\rangle\left(m \in \boldsymbol{Z}_{\geq 0}\right)$. We denote by $K^{(i)}(\alpha)$ the linear operator acting on the $i$-component $\mathscr{F}^{(i)}$ of $\mathscr{F}^{[0, M]}$ as $K(\alpha)$. Then it holds that

$$
\left[L^{(i)}(z ; s),\left(\begin{array}{cc}
1 & 0 \\
0 & \alpha
\end{array}\right) K^{(i)}(\alpha)\right]=0
$$

in $\mathscr{B}^{[0, M]} \otimes \operatorname{End}\left(\boldsymbol{C}^{2}\right)$. Using this property and the relation

$$
\tilde{L}^{(i)}(u ; s)=\frac{1}{1-s u}\left(\begin{array}{cc}
1 & 0 \\
0 & (-s u)^{-1}
\end{array}\right) L^{(i)}(-s u ; s)\left(\begin{array}{cc}
1 & 0 \\
0 & u
\end{array}\right),
$$

we find that

$$
\begin{aligned}
\tilde{C}^{[0, M]}(u ; s)= & (-s u)^{-1}(-s(1-s u))^{-(M+1)} \prod_{1 \leq i \leq k} K^{(i)}\left((-s)^{-(M+1-i)}\right) \\
& \times C^{[0, M]}(-s u ; s) \prod_{1 \leq i \leq k} K^{(i)}\left((-s)^{M+1-i}\right),
\end{aligned}
$$

where $C^{[0, M]}(z ; s)$ is the operator defined by the right hand side of (5.3) with $M^{\prime}=0$ and $L^{(i)}(z)$ replaced by $L^{(i)}(z ; s)(0 \leq i \leq M)$. Substitute this expression of $\tilde{C}^{[0, M]}(u ; s)$ into (5.6) and change the variables $u_{i}$ to $-z_{i} / s(0 \leq i \leq k)$. Then we see that

$$
\begin{aligned}
& F_{\vec{x}}\left(-z_{1} / s, \ldots,-z_{k} / s\right) \\
& \quad=\frac{(-s)^{\sum_{i=1}^{k} x_{i}}}{\prod_{i=1}^{k} z_{i}\left(1+z_{i}\right)^{M+1}}\left\langle\prod_{1 \leq i \leq k} C^{[0, M]}\left(u_{i} ; s\right) \prod_{1 \leq i \leq k} \beta_{x_{i}}^{*}\right\rangle_{[0, M]} .
\end{aligned}
$$

Now we can obtain the formula (5.5) as follows. From the definition of $F_{\vec{x}}$, the left hand side of $(5.5)$ is equal to the limit

$$
\lim _{s \rightarrow 0}\left\{\left(1-q^{2}\right)^{-k}(-s)^{-\sum_{i=1}^{k} x_{i}} \prod_{i=1}^{k}\left(1+z_{i}\right) F_{\vec{x}}\left(-z_{1} / s, \ldots,-z_{k} / s\right)\right\} .
$$

Using the formula (5.7) and $C^{[0, M]}(z ; 0)=C^{[0, M]}(z)$, we find that the limit is equal to the right hand side of (5.5). 
Remark 5.8. In [8] Motegi and Sakai prove a formula which represents the $\beta$-Grothendieck polynomial [3] as the wavefunction of the non-Hermitian phase model (here $\beta$ is a parameter and not the $q$-deformed boson). At the $K$-theoretical point $\beta=-1$, their formula is equivalent to the equality (5.5) with $q=0$.

\subsection{Common properties of $h_{\vec{z}}^{\vec{\mu}}$ and $\psi_{\vec{z}}^{\vec{\mu}}$}

The proof of Theorem 5.4 will consist of establishing that the functions $h_{\vec{z}}^{\vec{\mu}}$ and $\psi_{\vec{z}}^{\vec{\mu}}$ share three common properties. First we show two of them.

Proposition 5.9. Both $h_{\vec{z}}^{\vec{\mu}}$ and $\psi_{\vec{z}}^{\vec{\mu}}$ have the shift invariance

$$
F_{\vec{z}}^{\vec{\mu}}\left(x_{1}+c, \ldots, x_{k}+c\right)=\prod_{i=1}^{k}\left(\frac{z_{i}}{1+z_{i}}\right)^{c} F_{\vec{z}}^{\vec{\mu}}\left(x_{1}, \ldots, x_{k}\right) \quad\left(\vec{x} \in L_{+}, c \in \boldsymbol{Z}\right) .
$$

Proof. It follows from the definition of $h_{\vec{z}}^{\vec{\mu}}$ and $\psi_{\vec{z}}^{\vec{\mu}}$.

Proposition 5.10. Both $h_{\vec{z}}^{\vec{\mu}}$ and $\psi_{\vec{z}}^{\vec{\mu}}$ satisfy the following symmetry conditions.

(i) If $\vec{\mu} \in I_{k_{1}, \ldots, k_{r}}$ satisfies $\mu_{i}=\mu_{i+1}$, then $F_{\ldots, \ldots, z_{i}, z_{i+1}, \ldots}^{\ldots}=F_{\ldots, \ldots, z_{i+1}, z_{i}, \ldots}$.

(ii) If $\vec{\mu} \in I_{k_{1}, \ldots, k_{r}}$ satisfies $\mu_{i}<\mu_{i+1}$, then

$$
q F_{\ldots, z_{i}, z_{i+1}, \ldots}^{\ldots, \mu_{i+1}, \ldots}=f\left(z_{i}, z_{i+1}\right) F_{\ldots, z_{i+1}, z_{i}, \ldots}^{\cdots, \mu_{i+1}, \mu_{i}, \ldots}-g\left(z_{i+1}, z_{i}\right) F_{\ldots, z_{i}, z_{i+1}, \ldots}^{\ldots, \mu_{i+1}, \mu_{i}, \ldots},
$$

where $f(z, w)$ and $g(z, w)$ are defined by (3.3).

Proof. It follows from Lemma 3.1 and Proposition 4.3.

Proposition 5.9 and Proposition 5.10 imply that, in order to prove Theorem 5.4, it suffices to show $h_{\vec{z}}^{\vec{\mu}}(x)=\psi_{\vec{z}}^{\vec{\mu}}(x)$ for $\vec{\mu}=\left(r^{k_{r}}, \ldots, 1^{k_{1}}\right)$ and $x \in L_{k}^{+}$such that $x_{k}=1$. Note that

$$
h_{z}^{a}(1)=\frac{z}{1+z} u_{a}=\psi_{z}^{a}(1)
$$

for $z \in C$ and $1 \leq a \leq r$. Hence Theorem 5.4 follows from the recurrence relation below.

Theorem 5.11. Suppose that $x_{1} \geq \cdots \geq x_{k-1} \geq 1$ and $\vec{z} \in C^{k}$. Both $h_{\vec{z}}^{\vec{\mu}}$ and $\psi_{\vec{z}}^{\vec{\mu}}$ satisfy the following relation.

$$
\begin{aligned}
F_{\vec{z}}^{r^{k_{r}}, \ldots, 1^{k_{1}}}\left(x_{1}, \ldots, x_{k-1}, 1\right) & \\
& =\sum_{a=1}^{r} q^{\sum_{p=1}^{a-1} k_{p}} \sum_{t=0}^{r-a} \sum_{r \geq p(t)>\ldots>p(1)>p(0)=a} \sum_{\ell(t) \in J_{p(t)}} \ldots \sum_{\ell(0) \in J_{p(0)}} \frac{z_{\ell(t)}}{1+z_{\ell(t)}}
\end{aligned}
$$




$$
\begin{aligned}
& \times \prod_{s=1}^{t} g\left(z_{\ell(s)}, z_{\ell(s-1)}\right) \prod_{s=0}^{t}\left(\prod_{\substack{i \in J_{p(s+1)-1} \cup \ldots \cup J_{p(s)} \\
i \neq \ell(s)}} f\left(z_{i}, z_{\ell(s)}\right)\right) \\
& \times F_{\vec{z}(\ell(t), \ldots, \ell(0))}^{r^{k_{r}}, \ldots, a^{k_{a}-1}, \ldots, 1^{k_{1}}}\left(x_{1}, \ldots, x_{k-1}\right) \otimes u_{a},
\end{aligned}
$$

where we set $J_{p(t+1)-1}=J_{r}$ by agreement in the rightmost product, $J_{p}(1 \leq p \leq r)$ is given by

$$
J_{p}=\left\{i \in \boldsymbol{Z} \mid k_{r}+\cdots+k_{p+1}<i \leq k_{r}+\cdots+k_{p}\right\},
$$

and $\vec{z}(\ell(t), \ldots, \ell(0)) \in C^{k-1}$ is defined by

$$
\vec{z}(\ell(t), \ldots, \ell(0))_{i}= \begin{cases}z_{i} & (1 \leq i<\ell(0), i \neq \ell(t), \ldots, \ell(1)) \\ z_{\ell(s-1)} & (i=\ell(s), 1 \leq s \leq t) \\ z_{i+1} & (\ell(0) \leq i \leq k-1)\end{cases}
$$

Example 5.12. The $r=1$ case of the recurrence relation (5.8) reads as

$$
F_{\vec{z}}^{1^{k}}\left(x_{1}, \ldots, x_{k-1}, 1\right)=\sum_{\ell=1}^{k} \frac{z_{\ell}}{1+z_{\ell}} \prod_{\substack{0<i \leq k \\ i \neq \ell}} f\left(z_{i}, z_{\ell}\right) F_{\vec{z}(\ell)}^{1^{k-1}}\left(x_{1}, \ldots, x_{k-1}\right) \otimes u_{1},
$$

where $\vec{z}(\ell)=\left(z_{1}, \ldots, z_{\ell-1}, z_{\ell+1}, \ldots, z_{k}\right)$. In the case of $r=2$, it reads as follows:

$$
\begin{aligned}
F_{\vec{z}}^{2^{k_{2}}, 1^{k_{1}}} & \left(x_{1}, \ldots, x_{k-1}, 1\right) \\
= & \sum_{k_{2}<\ell \leq k} \frac{z_{\ell}}{1+z_{\ell}} \prod_{\substack{0<i \leq k \\
i \neq \ell}} f\left(z_{i}, z_{\ell}\right) F_{\vec{z}(\ell)}^{2^{k_{2}}, 1^{k_{1}-1}}\left(x_{1}, \ldots, x_{k-1}\right) \otimes u_{1} \\
& +\sum_{\substack{0<\ell_{1} \leq k_{2} \\
k_{2}<\ell_{0} \leq k}} \frac{z_{\ell_{1}}}{1+z_{\ell_{1}}} g\left(z_{\ell_{1}}, z_{\ell_{0}}\right) \prod_{\substack{0<i \leq k_{2} \\
i \neq \ell_{1}}} f\left(z_{i}, z_{\ell_{1}}\right) \\
& \times \prod_{\substack{k_{2}<i \leq k \\
i \neq \ell_{0}}} f\left(z_{i}, z_{\ell_{0}}\right) F_{\vec{z}\left(\ell_{1}, \ell_{0}\right)}^{2^{k_{2}}, 1_{1}^{k_{1}-1}}\left(x_{1}, \ldots, x_{k-1}\right) \otimes u_{1} \\
& +q^{k_{1}} \sum_{0<\ell \leq k_{2}} \frac{z_{\ell}}{1+z_{\ell}} \prod_{\substack{0<i \leq k_{2} \\
i \neq \ell}} f\left(z_{i}, z_{\ell}\right) F_{\vec{z}(\ell)}^{2^{k_{2}-1}, 1^{k_{1}}}\left(x_{1}, \ldots, x_{k-1}\right) \otimes u_{2},
\end{aligned}
$$

where

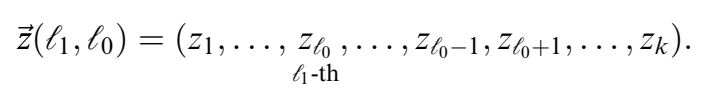


In the rest of this paper we prove the recurrence relation (5.8) for $h_{\vec{z}}^{\vec{\mu}}$ and $\psi_{\vec{z}}^{\vec{\mu}}$ successively.

\subsection{Proof for $h_{\vec{z}}^{\vec{\mu}}$}

First we prove the relation (5.8) for $h_{\vec{z}}^{\vec{\mu}}$. For that purpose we will show some relations on the space $U_{0} \otimes\left(U^{\otimes m}\right)$, where $m$ is a positive integer and $U_{0}$ is a copy of $U$ which we regard as the zeroth component. Then we set $R_{i}=\left(1^{\otimes i}\right) \otimes R \otimes\left(1^{\otimes(m-i-1)}\right) \in \operatorname{End}\left(U_{0} \otimes\left(U^{\otimes m}\right)\right)$ and define $Y_{i}(z, w)$ by $(3.4)$ for $0 \leq i<m$.

Let $m$ be a positive integer. For an interval $J$ included in $[1, m]$ (resp. $[2, m])$ and $\ell \in J$, we define the operator $Z_{\ell}^{J}(\vec{z}) \quad\left(\vec{z} \in C^{m}\right)$ acting on $U_{0} \otimes\left(U^{\otimes m}\right)$ (resp. $U^{\otimes m}$ ) by

$$
Z_{\ell}^{J}(\vec{z})=\prod_{\substack{i \in J \\ i<\ell}} f\left(z_{\ell}, z_{i}\right) \prod_{\substack{i \in J \\ i>\ell}} f\left(z_{i}, z_{\ell}\right) \prod_{\substack{i \in J \\ i<\ell}}^{\curvearrowleft} Y_{i-1}\left(z_{\ell}, z_{i}\right) .
$$

Hereafter we set

$$
u\left(v_{1}, \ldots, v_{m}\right)=u_{v_{1}} \otimes \cdots \otimes u_{v_{m}}
$$

for simplicity.

Lemma 5.13. Suppose that $r \geq b>a \geq 1$ and $m \geq 1$. Then it holds that

$$
\prod_{1 \leq i \leq m}^{\curvearrowleft}\left(f\left(w, z_{i}\right) Y_{i-1}\left(w, z_{i}\right)\right) u\left(b, a^{m}\right)=q^{m} u\left(a^{m}, b\right)+\sum_{\ell=1}^{m} g\left(w, z_{\ell}\right) Z_{\ell}^{[1, m]}(\vec{z}) u\left(b, a^{m}\right)
$$

on $U_{0} \otimes\left(U^{\otimes m}\right)$.

Proof. The proof is by induction on $m$. When $m=1$ it follows from the definition of $Y_{0}(w, z)$. Suppose that it holds for a positive integer $m$. We have

$$
\begin{aligned}
& \prod_{1 \leq i \leq m+1}^{\curvearrowleft}\left(f\left(w, z_{i}\right) Y_{i-1}\left(w, z_{i}\right)\right) u\left(b, a^{m+1}\right) \\
& =\prod_{2 \leq i \leq m+1}^{\curvearrowleft}\left(f\left(w, z_{i}\right) Y_{i-1}\left(w, z_{i}\right)\right)\left\{q u\left(a, b, a^{m}\right)+g\left(w, z_{1}\right) u\left(b, a^{m+1}\right)\right\} \\
& =q \prod_{2 \leq i \leq m+1}^{\curvearrowleft}\left(f\left(w, z_{i}\right) Y_{i-1}\left(w, z_{i}\right)\right) u\left(a, b, a^{m}\right) \\
& \quad+g\left(w, z_{1}\right) \prod_{i=2}^{m+1} f\left(z_{i}, w\right) u\left(b, a^{m+1}\right) .
\end{aligned}
$$


By the induction hypothesis the first term in the right hand side is equal to

$$
q\left\{q^{m} u\left(a^{m}, b\right)+\sum_{\ell=2}^{m+1} g\left(w, z_{\ell}\right) Z_{\ell}^{[2, m+1]}(\vec{z}) u\left(a, b, a^{m}\right)\right\} .
$$

Now use the equality

$$
u\left(a, b, a^{m}\right)=q^{-1}\left\{f\left(z_{\ell}, z_{1}\right) Y_{0}\left(z_{\ell}, z_{1}\right)-g\left(z_{\ell}, z_{1}\right)\right\} u\left(b, a^{m+1}\right)
$$

to substitute for the second term of (5.12), and we see that the right hand side of $(5.11)$ is equal to

$$
\begin{aligned}
& q^{m+1} u\left(a^{m}, b\right)+\sum_{\ell=2}^{m+1} g\left(w, z_{\ell}\right) Z_{\ell}^{[1, m+1]}(\vec{z}) u\left(b, a^{m+1}\right) \\
& \quad+\left\{g\left(w, z_{1}\right) \prod_{i=2}^{m+1} f\left(z_{i}, w\right)-\sum_{\ell=2}^{m+1} g\left(w, z_{\ell}\right) g\left(z_{\ell}, z_{1}\right) \prod_{\substack{i=2 \\
i \neq \ell}}^{m+1} f\left(z_{i}, z_{\ell}\right)\right\} u\left(b, a^{m+1}\right) .
\end{aligned}
$$

The coefficient of the third term above is equal to $g\left(w, z_{1}\right) \prod_{i=2}^{m+1} f\left(z_{i}, z_{1}\right)$. Thus we obtain the desired equality for $m+1$.

Lemma 5.14. Suppose that $r \geq b>a \geq 1$ and $k_{a}, k_{a-1}, \ldots, k_{1}$ are nonnegative integers. Define the intervals $J_{p}(1 \leq p \leq a)$ by

$$
J_{p}=\left\{i \in \boldsymbol{Z} \mid k_{a}+\cdots+k_{p+1}<i \leq k_{a}+\cdots+k_{p}\right\} .
$$

Then it holds that

$$
\begin{aligned}
q^{\sum_{p=2}^{a} k_{p}} & \sum_{\ell(1) \in J_{1}} g\left(w, z_{\ell(1)}\right) Z_{\ell(1)}^{J_{1}}(\vec{z}) u\left(a^{k_{a}}, \ldots, 2^{k_{2}}, b, 1^{k_{1}}\right) \\
= & \sum_{t=1}^{a} \sum_{a \geq p(t)>\cdots>p(2)>p(1)=1} \sum_{\ell(t) \in J_{p(t)}} \ldots \sum_{\ell(1) \in J_{p(1)}}(-1)^{t-1} \prod_{s=1}^{t} g\left(z_{\ell(s-1)}, z_{\ell(s)}\right) \\
& \times \prod_{1 \leq s \leq t}^{\curvearrowleft} Z_{\ell(s)}^{J_{p(s+1)-1} \cup \cdots \cup J_{p(s)}}(\vec{z}) u\left(b, a^{k_{a}}, \ldots, 1^{k_{1}}\right)
\end{aligned}
$$

on $U_{0} \otimes\left(U^{\otimes\left(k_{a}+\cdots+k_{1}\right)}\right)$, where we set $z_{\ell(0)}=w$ and $J_{p(t+1)-1}=J_{a}$ in the right hand side.

Proof. We proceed by induction on $a$. If $a=1$, it is trivial. Suppose that it holds for an integer $a$ such that $b-2 \geq a \geq 1$. By the induction hypothesis we have 


$$
\begin{aligned}
q^{\sum_{p=2}^{a+1} k_{p}} & \sum_{\ell(1) \in J_{1}} g\left(w, z_{\ell(1)}\right) Z_{\ell(1)}^{J_{1}}(\vec{z}) u\left((a+1)^{k_{a+1}}, a^{k_{a}}, \ldots, 2^{k_{2}}, b, 1^{k_{1}}\right) \\
= & q^{k_{a+1}} \sum_{t=1}^{a} \sum_{a \geq p(t)>\ldots>p(2)>p(1)=1} \sum_{\ell(t) \in J_{p(t)}} \ldots \sum_{\ell(1) \in J_{p(1)}}(-1)^{t-1} \prod_{s=1}^{t} g\left(z_{\ell(s-1)}, z_{\ell(s)}\right) \\
& \times \prod_{1 \leq s \leq t}^{\curvearrowleft} Z_{\ell(s)}^{J_{p(s+1)-1} \cup \cdots \cup J_{p(s)}}(\vec{z}) u\left((a+1)^{k_{a+1}}, b, a^{k_{a}}, \ldots, 1^{k_{1}}\right) .
\end{aligned}
$$

The vector $q^{k_{a+1}} u\left((a+1)^{k_{a+1}}, b, a^{k_{a}}, \ldots, 1^{k_{1}}\right)$ in the right hand side is equal to

$$
\begin{aligned}
& \left\{\prod_{i \in J_{a+1}}^{\curvearrowleft}\left(f\left(z_{\ell(t)}, z_{i}\right) Y_{i-1}\left(z_{\ell(t)}, z_{i}\right)\right)-\sum_{\ell(t+1) \in J_{a+1}} g\left(z_{\ell(t)}, z_{\ell(t+1)}\right) Z_{\ell(t+1)}^{J_{a+1}}(\vec{z})\right. \\
& \quad \times u\left(b,(a+1)^{k_{a+1}}, a^{k_{a}}, \ldots, 1^{k_{1}}\right)
\end{aligned}
$$

because of Lemma 5.13. Substituting it we see that the equality to prove is also true for $a+1$.

Combining Lemma 5.13 and Lemma 5.14, we obtain the following formula.

Corollary 5.15. Suppose that $r>a \geq 1$ and $k_{a}, k_{a-1}, \ldots, k_{1}$ are nonnegative integers. Define the intervals $J_{p}(1 \leq p \leq a)$ by (5.13). Then it holds that

$$
\begin{aligned}
q^{\sum_{p=2}^{a} k_{p}} & \prod_{i \in J_{1}}^{\curvearrowleft}\left(f_{i}\left(w, z_{i}\right) Y_{i-1}\left(w, z_{i}\right)\right) u\left(a^{k_{a}}, \ldots, 2^{k_{2}}, a+1,1^{k_{1}}\right) \\
= & q^{\sum_{p=1}^{a} k_{p}} u\left(a^{k_{a}}, \ldots, 1^{k_{1}}, a+1\right) \\
& +\sum_{t=1}^{a} \sum_{a \geq p(t)>\ldots>p(2)>p(1)=1} \sum_{\ell(t) \in J_{p(t)}} \ldots \sum_{\ell(1) \in J_{p(1)}}(-1)^{t-1} \prod_{s=1}^{t} g\left(z_{\ell(s-1)}, z_{\ell(s)}\right) \\
& \times \prod_{1 \leq s \leq t}^{\curvearrowleft} Z_{\ell(s)}^{J_{p(s+1)-1} \cup \cdots \cup J_{p(s)}(\vec{z})} u\left(a+1, a^{k_{a}}, \ldots, 1^{k_{1}}\right) .
\end{aligned}
$$

on $U_{0} \otimes\left(U^{\otimes\left(k_{a}+\cdots+k_{1}\right)}\right)$, where we set $z_{\ell(0)}=w$ and $J_{p(t+1)-1}=J_{a}$ in the right hand side.

Proposition 5.16. Suppose that $r>a \geq 1$ and $k_{a}, k_{a-1}, \ldots, k_{1}$ are nonnegative integers. Define the intervals $J_{p}(1 \leq p \leq a)$ by (5.13). Then it holds that 


$$
\begin{aligned}
& \prod_{1 \leq i \leq k_{a}+\cdots+k_{1}}^{\curvearrowleft}\left(f\left(w, z_{i}\right) Y_{i-1}(w, z)\right) u\left(a+1, a^{k_{a}}, \ldots, 1^{k_{1}}\right) \\
& =q^{\sum_{p=1}^{a} k_{p}} u\left(a^{k_{a}}, \ldots, 1^{k_{1}}, a+1\right) \\
& \quad+\sum_{t=1}^{a} \sum_{a \geq p(t)>\cdots>p(1) \geq 1} q^{\sum_{b=1}^{p(1)-1} k_{b}} \sum_{\ell(t) \in J_{p(t)}} \ldots \sum_{\ell(1) \in J_{p(1)}} \prod_{s=1}^{t} g\left(z_{\ell(s+1)}, z_{\ell(s)}\right) \\
& \quad \times \prod_{1 \leq s \leq t}^{\curvearrowleft} Z_{\ell(s)}^{J_{p(s+1)-1} \cup \cdots \cup J_{p(s)}}(\vec{z}) u\left(a+1, a^{k_{a}}, \ldots, p(1)^{k_{p(1)}-1}, \ldots, 1^{k_{1}}, p(1)\right) .
\end{aligned}
$$

on $U_{0} \otimes\left(U^{\otimes\left(k_{a}+\cdots+k_{1}\right)}\right)$, where we set $z_{\ell(0)}=w$ and $J_{p(t+1)-1}=J_{a}$ in the right hand side.

Proof. The proof is by induction on $a$. If $a=1$, it follows from Lemma 5.13. Suppose that it holds for an integer $a$ such that $r-1>a \geq 1$. Using the induction hypothesis we see that the left hand side of (5.14) with $a$ replaced by $a+1$ is equal to the image of the vector

$$
\begin{aligned}
& q^{\sum_{p=2}^{a+1} k_{p}}\left[u\left((a+1)^{k_{a+1}}, \ldots, 2^{k_{1}}, a+2,1^{k_{1}}\right)\right. \\
& \quad+\sum_{t=1}^{a} \sum_{a+1 \geq p(t)>\cdots>p(1) \geq 2} q^{\sum_{b=2}^{p(1)-1} k_{b}} \sum_{\ell(t) \in J_{p(t)}} \ldots \sum_{\ell(1) \in J_{p(1)}} \prod_{s=1}^{t} g\left(z_{\ell(s+1)}, z_{\ell(s)}\right) \\
& \left.\quad \times \prod_{1 \leq s \leq t}^{\curvearrowleft} Z_{\ell(s)}^{J_{p(s+1)-1} \cup \cdots \cup J_{p(s)}}(\vec{z}) u\left(a+2,(a+1)^{k_{a}}, \ldots, p(1)^{k_{p(1)}-1}, \ldots, 2^{k_{2}}, p(1), 1^{k_{1}}\right)\right],
\end{aligned}
$$

where $z_{\ell(t+1)}=w$ and $J_{p(t+1)-1}=J_{a+1}$, under the operator $\prod_{i \in J_{1}}^{\curvearrowleft}\left(f\left(w, z_{i}\right)\right.$. $\left.Y_{i-1}(w, z)\right)$. Now use Corollary 5.15 to calculate the image, and we see that it is equal to the sum of the following four vectors:

$$
\begin{aligned}
X_{I}= & q^{\sum_{p=1}^{a+1} k_{a}} u\left((a+1)^{k_{a+1}}, \ldots, 1^{k_{1}}, a+2\right), \\
X_{I I}= & \sum_{t=1}^{a+1} \sum_{a+1 \geq p(t)>\cdots>p(2)>p(1)=1} \sum_{\ell(t) \in J_{p(t)}} \ldots \sum_{\ell(1) \in J_{p(1)}}(-1)^{t-1} g\left(w, z_{\ell(1)}\right) \\
& \times \prod_{s=1}^{t-1} g\left(z_{\ell(s)}, z_{\ell(s+1)}\right) \prod_{1 \leq s \leq t}^{\curvearrowleft} Z_{\ell(s)}^{J_{p(s+1)-1} \cup \cdots \cup J_{p(s)}(\vec{z})} u\left(a+2,(a+1)^{k_{a+1}}, \ldots, 1^{k_{1}}\right),
\end{aligned}
$$




$$
\begin{aligned}
X_{I I I}= & \sum_{t=1}^{a} \sum_{a+1 \geq p(t)>\cdots>p(1) \geq 2} q^{\sum_{p=1}^{p(1)-1} k_{p}} \sum_{\ell(t) \in J_{p(t)}} \ldots \sum_{\ell(1) \in J_{p(1)}} g\left(w, z_{\ell(t)}\right) \prod_{s=1}^{t-1} g\left(z_{\ell(s+1)}, z_{\ell(s)}\right) \\
& \times \prod_{1 \leq s \leq t}^{\curvearrowleft} Z_{\ell(s)}^{J_{p(s+1)-1} \cup \cdots \cup J_{p(s)}}(\vec{z}) u\left(a+2,(a+1)^{k_{a+1}}, \ldots, p(1)^{k_{p(1)}-1}, \ldots, 1^{k_{1}}, p(1)\right),
\end{aligned}
$$

where $J_{p(t+1)-1}=J_{a+1}$, and

$$
\begin{aligned}
X_{I V}= & \sum_{t=2}^{a+1} \sum_{a+1 \geq p(t)>\cdots>p(2)>p(1)=1} \sum_{\ell(t) \in J_{p(t)}} \ldots \sum_{\ell(1) \in J_{p(1)}} K_{t}\left(w ; z_{\ell(1)}, \ldots, z_{\ell(t)}\right) \\
& \times \prod_{1 \leq s \leq t}^{\curvearrowleft} Z_{\ell(s)}^{J_{p(s+1)-1} \cup \cdots \cup J_{p(s)}(\vec{z})} u\left(a+2,(a+1)^{k_{a+1}}, \ldots, 1^{k_{1}}\right),
\end{aligned}
$$

where

$$
\begin{aligned}
& K_{t}\left(w ; z_{\ell(1)}, \ldots, z_{\ell(t)}\right) \\
& \quad=\sum_{s=0}^{t-2} g\left(w, z_{\ell(1)}\right) g\left(w, z_{\ell(t)}\right) \prod_{i=1}^{s} g\left(z_{\ell(i)}, z_{\ell(i+1)}\right) \prod_{i=s+2}^{t-1} g\left(z_{\ell(i+1)}, z_{\ell(i)}\right) .
\end{aligned}
$$

From the definition of $g$, we find that

$$
K_{t}\left(w ; z_{\ell(1)}, \ldots, z_{\ell(t)}\right)=\frac{g\left(w, z_{\ell(1)}\right) g\left(w, z_{\ell(t)}\right)}{g\left(z_{\ell(t)}, z_{\ell(1)}\right)} \prod_{s=1}^{t-1} g\left(z_{\ell(s+1)}, z_{\ell(s)}\right)
$$

for $t \geq 2$. Substitute it for $X_{I V}$ and add $X_{I I}$ and $X_{I V}$ using

$$
\begin{aligned}
& (-1)^{t-1} \prod_{s=1}^{t-1} g\left(z_{\ell(s)}, z_{\ell(s+1)}\right)+\frac{g\left(w, z_{\ell(t)}\right)}{g\left(z_{\ell(t)}, z_{\ell(1)}\right)} \prod_{s=1}^{t-1} g\left(z_{\ell(s+1)}, z_{\ell(s)}\right) \\
& =\frac{g\left(w, z_{\ell(t)}\right)}{g\left(w, z_{\ell(1)}\right)} \prod_{s=1}^{t-1} g\left(z_{\ell(s+1)}, z_{\ell(s)}\right) .
\end{aligned}
$$

As a result we get the desired equality for $a+1$.

Now let us prove the recurrence relation (5.8) for $h_{\vec{z}}^{\vec{\mu}}$.

Proof of Theorem 5.11 for $h_{\vec{z}}^{\vec{\mu}}$. Set $J_{p}=\left\{i \in \boldsymbol{Z} \mid k_{r}+\cdots+k_{p+1}<i \leq\right.$ $\left.k_{r}+\cdots+k_{p}\right\}$ for $1 \leq p \leq r$. In the definition of the value $h_{\vec{z}}^{r^{k_{r}}, \ldots, 1^{k_{1}}}\left(x_{1}, \ldots\right.$, $\left.x_{k-1}, 1\right)$, decompose the sum over $\tau \in \Xi_{k}$ into $k$ parts with $\tau^{-1}(k)=\ell$ $(1 \leq \ell \leq k)$. Change the running index $\tau$ to $\tau^{\prime}=\tau\left(\sigma_{\ell} \sigma_{\ell+1} \ldots \sigma_{k-1}\right)$, where $\sigma_{i}=(i, i+1)$ is the transposition. Then $\tau^{\prime}(k)=k$ and we can regard $\tau^{\prime}$ as an element of $\mathfrak{\Xi}_{k-1}$. Thus we see that 


$$
\begin{aligned}
h_{\vec{z}}^{r^{k_{r}, \ldots, 1^{k_{1}}}}\left(x_{1}, \ldots, x_{k-1}, 1\right) \\
=\prod_{1 \leq i<j \leq k} f\left(z_{i}, z_{j}\right) \sum_{p=1}^{r} \sum_{\ell \in J_{p}} \sum_{\tau^{\prime} \in \mathbb{S}_{k-1}} \frac{z_{\ell}}{1+z_{\ell}} \prod_{i=1}^{\ell-1}\left(\frac{z_{i}}{1+z_{i}}\right)^{x_{\tau^{\prime}(i)}} \\
\quad \times \prod_{i=\ell+1}^{k}\left(\frac{z_{i}}{1+z_{i}}\right)^{x_{\tau^{\prime}(i-1)}} \phi\left(\tau^{\prime} ; z_{1}, \ldots, z_{\ell-1}, z_{\ell+1}, \ldots, z_{k}\right) \\
\quad \times \phi\left(\sigma_{k-1} \ldots \sigma_{\ell} ; \vec{z}\right) u\left(r^{k_{r}}, \ldots, 1^{k_{1}}\right) .
\end{aligned}
$$

For $\ell \in J_{p}$ it holds that

$$
\phi\left(\sigma_{k-1} \ldots \sigma_{\ell} ; \vec{z}\right) u\left(r^{k_{r}}, \ldots, 1^{k_{1}}\right)=\prod_{\substack{i \in J_{p} \\ i>\ell}} \frac{f\left(z_{i}, z_{\ell}\right)}{f\left(z_{\ell}, z_{i}\right)} \prod_{i \in J_{p+1} \cup \cdots \cup J_{1}}^{\curvearrowleft} Y_{i-1}\left(z_{\ell}, z_{i}\right) u\left(r^{k_{r}}, \ldots, 1^{k_{1}}\right) .
$$

From Proposition 5.16, we have

$$
\begin{aligned}
& \prod_{i \in J_{p+1} \cup \cdots \cup J_{1}}^{\curvearrowleft} Y_{i-1}\left(z_{\ell}, z_{i}\right) u\left(r^{k_{r}}, \ldots, 1^{k_{1}}\right) \\
& =q^{\sum_{b=1}^{p-1} k_{b}} u\left(r^{k_{r}}, \ldots, p^{k_{p}-1}, \ldots, 1^{k_{1}}, p\right) \\
& \quad+\sum_{t=1}^{p-1} \sum_{p-1 \geq p(t-1)>\cdots>p(0) \geq 1} q^{\sum_{b=1}^{p(0)-1} k_{b}} \sum_{\ell(t-1) \in J_{p(t-1)}} \ldots \sum_{\ell(0) \in J_{p(0)}} \prod_{s=1}^{t} g\left(z_{\ell(s)}, z_{\ell(s-1)}\right) \\
& \quad \times \prod_{0 \leq s \leq t-1}^{\curvearrowleft} Z_{\ell(s)}^{J_{p(s+1)-1} \cup \cdots \cup J_{p(s)}}(\vec{z}) u\left(r^{k_{r}}, \ldots, p(0)^{k_{p(0)}-1}, \ldots, 1^{k_{1}}, p(0)\right),
\end{aligned}
$$

where $J_{p(t)-1}=J_{p-1}$. Thus we see that the right hand side of (5.15) is the sum of the following two terms:

$$
S_{I}=\sum_{a=1}^{r} q^{\sum_{b=1}^{a-1} k_{b}} \sum_{\ell \in J_{a}} \frac{z_{\ell}}{1+z_{\ell}} \prod_{\substack{i \in J_{r} \cup \ldots . \cup J_{a} \\ i \neq \ell}} f\left(z_{i}, z_{\ell}\right) h_{z_{1}, \ldots, z_{\ell-1}, z_{\ell+1}, \ldots, z_{k}}^{r^{k_{r}}, \ldots, a^{k_{a}-1}, \ldots, 1^{k_{1}}}\left(x_{1}, \ldots, x_{k-1}\right) \otimes u_{a}
$$

and

$$
\begin{aligned}
S_{I I}= & \prod_{1 \leq i<j \leq k} f\left(z_{i}, z_{j}\right) \\
& \times \sum_{a=1}^{r-1} q^{\sum_{b=1}^{a-1} k_{b}} \sum_{t=1}^{r-a} \sum_{r \geq p(t)>\cdots>p(1)>p(0)=a} \sum_{\ell(t) \in J_{p(t)}} \ldots \sum_{\ell(0) \in J_{p(0)}} \prod_{\substack{i \in J_{p(t)} \\
i>\ell(t)}} \frac{f\left(z_{i}, z_{\ell(t)}\right)}{f\left(z_{\ell(t)}, z_{i}\right)}
\end{aligned}
$$




$$
\begin{aligned}
& \times \sum_{\tau^{\prime} \in \mathbb{S}_{k-1}} \frac{z_{\ell(t)}}{1+z_{\ell(t)}} \prod_{i=1}^{\ell(t)-1}\left(\frac{z_{i}}{1+z_{i}}\right)^{x_{\tau^{\prime}(i)}} \prod_{i=\ell(t)+1}^{k}\left(\frac{z_{i}}{1+z_{i}}\right)^{x_{\tau^{\prime}(i-1)}} \prod_{s=1}^{t} g\left(z_{\ell(s)}, z_{\ell(s-1)}\right) \\
& \times \phi\left(\tau^{\prime} ; z_{1}, \ldots, z_{\ell(t)-1}, z_{\ell(t)+1}, \ldots, z_{k}\right) \prod_{0 \leq s \leq t-1}^{\curvearrowleft} Z_{\ell(s)}^{J_{p(s+1)-1} \cup \cdots \cup J_{p(s)}(\vec{z})} \\
& \times u\left(r^{k_{r}}, \ldots, a^{k_{a}-1}, \ldots, 1^{k_{1}}, a\right) .
\end{aligned}
$$

We rewrite $S_{I I}$ as follows. Lemma 3.1 implies that

$$
\begin{aligned}
& \prod_{1 \leq i<j \leq k} f\left(z_{i}, z_{j}\right) \prod_{\substack{i \in J_{p(t)} \\
i>\ell(t)}} \frac{f\left(z_{i}, z_{\ell(t)}\right)}{f\left(z_{\ell(t)}, z_{i}\right)} \phi\left(\tau^{\prime} ; z_{1}, \ldots, z_{\ell(t)-1}, z_{\ell(t)+1}, \ldots, z_{k}\right) \\
& \quad \times \prod_{0 \leq s \leq t-1}^{\curvearrowleft} Z_{\ell(s)}^{J_{p(s+1)-1} \cup \cdots \cup J_{p(s)}(\vec{z})} \\
& =\prod_{1 \leq i<j \leq k-1} f\left(w_{i}, w_{j}\right) \prod_{s=0}^{t}\left(\prod_{\substack{i \in J_{p(s+1)-1} \cup \ldots \cup J_{p(s)} \\
i \neq \ell(s)}} f\left(z_{i}, z_{\ell(s)}\right)\right) \phi(\tilde{\tau} ; \vec{w}),
\end{aligned}
$$

where $\tilde{\tau} \in \mathfrak{\Xi}_{k-1}$ and $\vec{w}=\left(w_{1}, \ldots, w_{k-1}\right)$ are defined by

$$
\tilde{\tau}=\tau^{\prime} \prod_{0 \leq s<t}^{\curvearrowleft}\left(\prod_{\substack{i \in J_{p(s)} \\ i<\ell(s)}}^{\curvearrowright} \sigma_{i-1}\right)
$$

and

$$
w_{i}= \begin{cases}z_{i} & \left(i \notin J_{p(t)} \cup \cdots \cup J_{p(0)} \text { and } i<k_{r}+\cdots+k_{p(0)}\right) \\ z_{i} & \left(i \in J_{p(s)} \text { and } i<\ell(s), 0 \leq s \leq t\right) \\ z_{i+1} & \left(i \in J_{p(s)} \text { and } i \geq \ell(s), 1 \leq s \leq t\right) \\ z_{\ell(s-1)} & \left(i=k_{r}+\cdots+k_{p(s)}, 1 \leq s \leq t\right) \\ z_{i+1} & \left(k_{r}+\cdots+k_{p(0)} \leq i \leq k-1\right) .\end{cases}
$$

Then it holds that

$$
\prod_{i=1}^{\ell(t)-1}\left(\frac{z_{i}}{1+z_{i}}\right)^{x_{\tau^{\prime}(i)}} \prod_{i=\ell(t)+1}^{k}\left(\frac{z_{i}}{1+z_{i}}\right)^{x_{\tau^{\prime}(i-1)}}=\prod_{i=1}^{k-1}\left(\frac{w_{i}}{1+w_{i}}\right)^{x_{\tilde{\tau}(i)}}
$$

Now change the running index $\tau^{\prime}$ to $\tilde{\tau}$ in $S_{I I}$ and we find that 


$$
\begin{aligned}
S_{I I}= & \sum_{a=1}^{r-1} q^{\sum_{b=1}^{a-1} k_{b}} \sum_{t=1}^{r-a} \sum_{r \geq p(t)>\cdots>p(1)>p(0)=a} \sum_{\ell(t) \in J_{p(t)}} \ldots \sum_{\ell(0) \in J_{p(0)}} \frac{z_{\ell(t)}}{1+z_{\ell(t)}} \\
& \times \prod_{s=1}^{t} g\left(z_{\ell(s)}, z_{\ell(s-1)}\right) \prod_{s=0}^{t}\left(\prod_{\substack{i \in J_{p(s+1)-1} \cup \ldots \cup J_{p(s)} \\
i \neq \ell(s)}} f\left(z_{i}, z_{\ell(s)}\right)\right) \\
& \times h_{\vec{w}}^{r^{k_{r}}, \ldots, a^{k_{a}-1}, \ldots, 1^{k_{1}}}\left(x_{1}, \ldots, x_{k-1}\right) \otimes u_{a},
\end{aligned}
$$

where $\vec{w}$ is defined by (5.16). Proposition 5.10 (1) implies that

$$
h_{\vec{w}}^{r^{k_{r}}, \ldots, a^{k_{a}-1}, \ldots, 1^{k_{1}}}\left(x_{1}, \ldots, x_{k-1}\right)=h_{\vec{z}(\ell(t), \ldots, \ell(0))}^{r^{k_{r}}, \ldots, a^{k_{a}-1}, \ldots, 1^{k_{1}}}\left(x_{1}, \ldots, x_{k-1}\right) .
$$

Now the term $S_{I}$ compensates for the lack of the terms in $S_{I I}$ with $t=0$, and we get the desired recurrence relation for $h_{\vec{z}}^{\vec{\mu}}$.

\subsection{Proof for $\psi_{\vec{z}}^{\vec{\mu}}$}

Next we show that the function $\psi_{\vec{z}}^{\vec{\mu}}$ also satisfies the recurrence relation (5.8). Fix $\vec{x}=\left(x_{1}, \ldots, x_{k}\right) \in L_{k}^{+}$and an integer $M$ such that $x_{k}=1$ and $M \geq x_{1}$. Then

$$
\begin{aligned}
& \psi_{\vec{z}}^{r^{k_{r}}, \ldots, 1^{k_{1}}}(\vec{x})=\left(1-q^{2}\right)^{-k} \sum_{\vec{v} \in I_{k_{1}, \ldots, k_{r}}} \prod_{i=1}^{k}\left(\frac{1}{1+z_{i}}\right)^{M} \\
& \times\left\langle\prod_{1 \leq i \leq k}^{\curvearrowright} C_{\mu_{i}}^{[1, M]}\left(z_{i}\right) \prod_{1 \leq i \leq k} \beta_{v_{i}, x_{i}}^{*}\right\rangle_{[1, M]} q^{t(\vec{v})-t(\vec{\mu})} u_{v_{1}} \otimes \cdots \otimes u_{v_{k}} .
\end{aligned}
$$

Hereafter we often omit the upper index $[1, M]$ of $A^{[1, M]}(z)$ and $C_{a}^{[1, M]}(z)$ $(1 \leq a \leq r)$.

Lemma 5.17. It holds that $C_{b}(z) \beta_{a, 1}^{*}=\beta_{a, 1}^{*} C_{b}(z)$ if $a<b$, and $C_{b}(z) \beta_{a, 1}^{*}=$ $q^{2} \beta_{a, 1}^{*} C_{b}(z)$ if $a>b$.

Proof. It follows from Proposition 4.4.

For $1 \leq a \leq r$ we set

$$
\tilde{A}_{a}(z)=q^{2 \sum_{p=a}^{r} N_{p, 1}} A^{[2, M]}(z) .
$$

Lemma 5.18. For $\left(z_{1}, \ldots, z_{m}\right) \in \boldsymbol{C}^{m}, w \in C$ and $1 \leq a \leq b \leq r$, it holds that 


$$
\begin{aligned}
\prod_{i=1}^{m} C_{b}\left(z_{i}\right) \cdot \tilde{A}_{a}(w) \equiv & \prod_{i=1}^{m} f\left(z_{i}, w\right) \tilde{A}_{a}(w) \prod_{i=1}^{m} C_{b}\left(z_{i}\right) \\
& +\sum_{\ell=1}^{m} \frac{z_{\ell}}{w} g\left(z_{\ell}, w\right) \prod_{\substack{i=1 \\
i \neq \ell}}^{m} f\left(z_{i}, z_{\ell}\right) \tilde{A}_{a}\left(z_{\ell}\right) C_{b}(w) \prod_{\substack{i=1 \\
i \neq \ell}}^{m} C_{a}\left(z_{i}\right)
\end{aligned}
$$

modulo the right ideal $\sum_{p=1}^{b-1} \beta_{p, 1}^{*} \mathscr{B}^{[1, M]}$.

Proof. The proof is by induction on $m$. For $m=1$ it follows from (4.8), (4.5) on the interval $[2, M]$ and the equality $f(z, w)+g(z, w)=q^{2}$. Suppose that it holds for a positive integer $m$. Now decompose $\prod_{i=1}^{m+1} C_{b}\left(z_{i}\right) \cdot \tilde{A}_{a}(w)=$ $C_{b}\left(z_{1}\right) \prod_{i=2}^{m+1} C_{b}\left(z_{i}\right) \cdot \tilde{A}_{a}(w)$ and calculate it using the induction hypothesis. Note that $\beta_{p, 1}^{*}(1 \leq p<b)$ commutes with $C_{b}(z)$ because of Lemma 5.17. Then we obtain the desired formula for $m+1$ by using the equality

$g\left(z_{1}, w\right) \prod_{i=2}^{m+1} f\left(z_{i}, w\right)+\sum_{\ell=2}^{m+1} g\left(z_{\ell}, w\right) g\left(z_{1}, z_{\ell}\right) \prod_{\substack{i=2 \\ i \neq \ell}}^{m+1} f\left(z_{i}, z_{\ell}\right)=g\left(z_{1}, w\right) \prod_{i=2}^{m+1} f\left(z_{i}, z_{1}\right)$

Proposition 5.19. For $\left(z_{1}, \ldots, z_{m}\right) \in \boldsymbol{C}^{m}$ and $1 \leq a \leq r$, it holds that

$$
\prod_{i=1}^{m} C_{a}\left(z_{i}\right) \cdot \beta_{a, 1}^{*} \equiv \beta_{a, 1}^{*} \prod_{i=1}^{m} C_{a}\left(z_{i}\right)+\left(1-q^{2}\right) \sum_{\ell=1}^{r} z_{\ell} \prod_{\substack{i=1 \\ i \neq \ell}}^{m} f\left(z_{i}, z_{\ell}\right) \cdot \tilde{A}_{a}\left(z_{\ell}\right) \prod_{\substack{i=1 \\ i \neq \ell}}^{m} C_{a}\left(z_{i}\right)
$$

modulo the right ideal $\sum_{p=1}^{a-1} \beta_{p, 1}^{*} \mathscr{B}^{[1, M]}$.

Proof. The recurrence relation (4.8) implies that

$$
C_{a}(z) \beta_{a, 1}^{*} \equiv \beta_{a, 1}^{*} C_{a}(z)+\left(1-q^{2}\right) z \tilde{A}_{a}(z) .
$$

Using the above relation repeatedly we find that

$$
\prod_{i=1}^{m} C_{a}\left(z_{i}\right) \cdot \beta_{a, 1}^{*} \equiv \beta_{a, 1}^{*} \prod_{i=1}^{m} C_{a}\left(z_{i}\right)+\left(1-q^{2}\right) \sum_{\ell=1}^{m} z_{\ell} \prod_{i=1}^{\ell-1} C_{a}\left(z_{i}\right) \cdot \tilde{A}_{a}\left(z_{\ell}\right) \prod_{i=\ell+1}^{m} C_{a}\left(z_{i}\right) .
$$

Move $\tilde{A}_{a}\left(z_{\ell}\right)$ in the right hand side to the left using Lemma 5.18. Then the equality

$$
\prod_{i=1}^{\ell-1} f\left(z_{i}, z_{\ell}\right)+\sum_{t=\ell+1}^{m} g\left(z_{\ell}, z_{t}\right) \prod_{\substack{i=1 \\ i \neq \ell}}^{t-1} f\left(z_{i}, z_{\ell}\right)=\prod_{\substack{i=1 \\ i \neq \ell}}^{m} f\left(z_{i}, z_{\ell}\right)
$$

implies the desired formula. 
Suppose that $1 \leq a<r$ and $m_{r}, m_{r-1}, \ldots, m_{a+1}$ are non-negative integers. Set

$$
C^{\left(m_{r}, m_{r-1}, \ldots, m_{a+1}\right)}(\vec{z})=\prod_{a+1 \leq p \leq r i=m_{r}+\cdots+m_{p+1}+1}^{\curvearrowleft} \prod_{p}\left(z_{i}\right)
$$

for $\vec{z} \in \boldsymbol{C}^{m_{r}+\cdots+m_{a+1}}$.

Lemma 5.20. Suppose that $1 \leq a<r$ and $k_{r}, k_{r-1}, \ldots, k_{a+1}$ are non-negative integers. For $a<p \leq r$, set $J_{p}=\left\{i \in \boldsymbol{Z} \mid k_{r}+\cdots+k_{p+1}<i \leq k_{r}+\cdots+k_{p}\right\}$. Then it holds that

$$
\begin{aligned}
C^{\left(k_{r}, k_{r-1}, \ldots, k_{a+1}\right)}(\vec{z}) \tilde{A}_{a}(w) & \left(\prod_{i \in J_{r} \cup \ldots \cup J_{a+1}} f\left(z_{i}, w\right)\right) \tilde{A}_{a}(w) C^{\left(k_{r}, k_{r-1}, \ldots, k_{a+1}\right)}(\vec{z}) \\
& +\sum_{t=1}^{r-a} \sum_{r \geq p(t)>\cdots>p(1)>a} \sum_{\ell(t) \in J_{p(t)}} \ldots \sum_{\ell(1) \in J_{p(1)}} \frac{z_{\ell(t)}}{w} \tilde{A}_{a}\left(z_{\ell(t)}\right) \prod_{s=1}^{t} g\left(z_{\ell(s)}, z_{\ell(s-1)}\right) \\
& \times \prod_{s=0}^{t}\left(\prod_{\substack{i \in J_{p(s+1)-1} \cup \ldots \cup J_{p(s)} \\
i \neq \ell(s)}} f\left(z_{i}, z_{\ell(s)}\right)\right) C^{\left(k_{r}, k_{r-1}, \ldots, k_{a+1}\right)}(\vec{z}(\ell(t), \ldots, \ell(1) ; w))
\end{aligned}
$$

modulo the right ideal $\sum_{p=1}^{r-1} \beta_{p, 1}^{*} \mathscr{B}^{[1, M]}$ for $\vec{z} \in C^{k_{r}+\cdots+k_{a+1}}$, where $z_{\ell(0)}=w$, $J_{p(t+1)-1}=J_{r}, J_{p(0)}=J_{a+1}$ and $\vec{z}(\ell(t), \ldots, \ell(1) ; w) \in C^{k_{r}+\cdots+k_{a+1}}$ is defined by

$$
\vec{z}(\ell(t), \ldots, \ell(1) ; w)_{i}= \begin{cases}z_{i} & (i \neq \ell(t), \ldots, \ell(1)) \\ z_{\ell(s-1)} & (i=\ell(s), 2 \leq s \leq t) \\ w & (i=\ell(1)) .\end{cases}
$$

Proof. By reverse induction on a using Lemma 5.18.

Proposition 5.21. Suppose that $1 \leq a \leq r$ and that $k_{r}, k_{r-1}, \ldots, k_{a+1} \in \boldsymbol{Z}_{\geq 0}$ and $k_{a} \in \boldsymbol{Z}_{\geq 1}$. Set $J_{p}=\left\{i \in \boldsymbol{Z} \mid k_{r}+\cdots+k_{p+1}<i \leq k_{r}+\cdots+k_{p}\right\} \quad(a \leq p \leq r)$. Then it holds that

$$
\begin{aligned}
{[1, M] } & \langle\operatorname{vac}| C^{\left(k_{r}, \ldots, k_{a}\right)}(\vec{z}) \beta_{a, 1}^{*} \\
& =\left(1-q^{2}\right) \sum_{t=0}^{r-a} \sum_{r \geq p(t)>\cdots>p(1)>p(0)=a} \sum_{\ell(t) \in J_{p(t)}} \ldots \sum_{\ell(0) \in J_{p(0)}} z_{\ell(t)}\left(1+z_{\ell(t)}\right)^{M-1}
\end{aligned}
$$




$$
\begin{aligned}
& \times \prod_{s=1}^{t} g\left(z_{\ell(s)}, z_{\ell(s-1)}\right) \prod_{s=0}^{t}\left(\prod_{\substack{i \in J_{p(s+1)-1} \cup \ldots \cup J_{p(s)} \\
i \neq \ell(s)}} f\left(z_{i}, z_{\ell(s)}\right)\right) \\
& \times_{[1, M]}\langle\operatorname{vac}| C^{\left(k_{r}, \ldots, k_{a+1}, k_{a}-1\right)}(\vec{z}(\ell(t), \ldots, \ell(0)))
\end{aligned}
$$

for $\vec{z} \in C^{k_{r}+\cdots+k_{a}}$, where $J_{p(t+1)-1}=J_{r}$ and $\vec{z}(\ell(t), \ldots, \ell(0)) \in C^{k_{r}+\cdots+k_{a}-1}$ is defined by (5.10).

Proof. Note that $[1, M]\langle\operatorname{vac}| \beta_{p, 1}^{*}=0$ for any $1 \leq p \leq r$. From Lemma 5.17 and Proposition 5.19 we see that the left hand side is equal to

$$
\left(1-q^{2}\right) \sum_{\ell \in J_{a}} z_{\ell} \prod_{i \in J_{a} \backslash\{\ell\}} f\left(z_{i}, z_{\ell}\right) \cdot[1, M]\langle\operatorname{vac}| C^{\left(k_{r}, \ldots, k_{a+1}\right)}(\vec{z}) \tilde{A}_{a}\left(z_{\ell}\right) \prod_{i \in J_{a} \backslash\{\ell\}} C_{a}\left(z_{i}\right) .
$$

Move $\tilde{A}_{a}\left(z_{\ell}\right)$ to the left by using Lemma 5.20. From (4.7) we have

$$
[1, M]\langle\operatorname{vac}| \tilde{A}(w)=(1+w)^{M-1}{ }_{[1, M]}\langle\operatorname{vac}| .
$$

Thus we obtain the desired formula.

Now we prove the recurrence relation (5.8) for $\psi_{\vec{z}}^{\vec{\mu}}$.

Proof of Theorem 5.11 for $\psi_{\vec{z}}^{\vec{\mu}}$. Set $\vec{\mu}=\left(r^{k_{r}}, \ldots, 1^{k_{1}}\right)$. Decompose the sum over $\vec{v}$ in the definition of $\psi_{\vec{z}} \overrightarrow{\vec{\mu}}\left(x_{1}, \ldots, x_{k-1}, 1\right)$ into $r$ parts with $v_{k}=a$ $(1 \leq a \leq r)$. Then Lemma 5.17 implies that

$$
\begin{aligned}
\psi_{\vec{z}}^{\vec{\mu}}\left(x_{1}, \ldots, x_{k-1}, 1\right) & \left(1-q^{2}\right)^{-k} \prod_{i=1}^{k} \frac{1}{\left(1+z_{i}\right)^{M}} \sum_{a=1}^{r} q^{2 \sum_{p=1}^{a-1} k_{p}} \\
& \times \sum_{\substack{\vec{v} \in I_{k_{r} . ., k_{1}} \\
v_{k}=a}}\left\langle C^{\left(k_{r}, \ldots, k_{a}\right)}(\vec{z}) \beta_{a, 1}^{*} C^{\left(k_{a-1}, \ldots, k_{1}\right)}(\overrightarrow{z[a]}) \prod_{i=1}^{k-1} \beta_{v_{i}, x_{i}}^{*}\right\rangle_{[1, M]} \\
& \times q^{t(\vec{\mu})-t(\vec{v})} u_{v_{1}} \otimes \cdots \otimes u_{v_{k-1}} \otimes u_{a},
\end{aligned}
$$

where $\overrightarrow{z[a]} \in C^{k_{a-1}+\cdots+k_{1}}$ is defined by $\overrightarrow{z[a]_{i}}=z_{i+k_{r}+\cdots+k_{a}}$. Now move $\beta_{a, 1}^{*}$ to the left using Proposition 5.21. Note that

$$
t(\vec{\mu})-t(\vec{v})=t\left(r^{k_{r}}, \ldots, a^{k_{1}-1}, \ldots, 1^{k_{1}}\right)-t\left(v_{1}, \ldots, v_{k-1}\right)-\sum_{b=1}^{a-1} k_{b}
$$

for $\vec{v} \in I_{k_{r}, \ldots, k_{1}}$ such that $v_{k}=a$. Thus we get the relation (5.8) for $\psi_{\vec{z}}^{\vec{\mu}}$. 


\title{
Acknowledgements
}

The research of the author is supported by JSPS KAKENHI Grant Number 26400106. The author thanks the referee for valuable comments.

\section{References}

[1] Borodin, A., On a family of symmetric rational functions, preprint, arXiv:1410.0976.

[2] Borodin, A., Corwin, I., Petrov, L. and Sasamoto, T., Spectral theory for the $q$-Boson particle system, Compos. Math., 151 (2015), no. 1, 1-67.

[3] Fomin, S. and Kirillov, A., Grothendieck polynomials and the Yang-Baxter equation, Formal power series and algebraic combinatorics (DIMACS), 183-189.

[4] Garbali, A., de Gier, A. and Wheeler, M., A new generalisation of Macdonald polynomials, preprint, arXiv:1605.07200.

[5] Jimbo, M., A $q$-analogue of $U(g l(N+1))$, Hecke algebra, and the Yang-Baxter equation, Lett. Math. Phys., 11 (1986), no. 3, 247-252.

[6] Kuan, J., A multi-species $\operatorname{ASEP}(q, j)$ and $q$-TAZRP with stochastic duality, preprint, arXiv:1605.00691.

[ 7 ] Kuniba, A., Mangazeev, V. V., Maruyama, S. and Okado, M., Stochastic $R$ matrix for $U_{q}\left(A_{n}^{(1)}\right)$, preprint, arXiv:1604.08304.

[8] Motegi, K. and Sakai, K., $K$-theoretic boson-fermion correspondence and melting crystals, J. Phys. A, 47 (2014), no. 44, 445202.

[ 9 ] Sasamoto, T. and Wadati, M., Exact results for one-dimensional totally asymmetric diffusion models, J. Phys. A, 31 (1998), no. 28, 6057-6071.

[10] Takeyama, Y., Algebraic construction of multi-species $q$-Boson system, preprint, arXiv:1507.02033.

[11] Tracy, C. and Widom, H., On the asymmetric simple exclusion process with multiple species, J. Stat. Phys., 150 (2013), no. 3, 457-470.

\author{
nuna adreso: \\ Division of Mathematics \\ Faculty of Pure and Applied Sciences \\ University of Tsukuba \\ Tsukuba, 305-8571 \\ Japan \\ E-mail: takeyama@math.tsukuba.ac.jp
}

(Recevita la 21-an de junio, 2016)

(Revizita la 9-an de decembro, 2016) 